\title{
APORTACIÓN AL CONOCIMIENTO DE LAS DIATOMEAS BENTÓNICAS DE LAS AGUAS CORRIENTES SALMANTINAS
}

\author{
Ramiro GARCÍA RÍO, Francisca GALLEGO MARTÍN \\ y $\mathrm{M}^{\mathrm{a}}$ Angeles SÁNCHEZ ANTA
}

\begin{abstract}
RESUMEN. Aportación al conocimiento de las diatomeas bentónicas de las aguas corrientes salmantinas. En este trabajo se da a conocer el primer catálogo florístico y fotográfico de las diatomeas que pueblan diversos ríos y arroyos salmantinos. Este catálogo consta de 197 táxones pertenecientes a 160 especies. Entre ellos, 14 son posibles novedades para la flora algal española. Asimismo, se ofrecen datos obtenidos de varios factores físico-químicos del medio estudiado.
\end{abstract}

Palabras clave. Diatomeas, algas, fitobentos, aguas corrientes, Salamanca, España.

SUMMARY. Contribution to knowledge on bentonic diatoms in the fresh water of the province of Salamanca. This paper presents the first floristic and photographic catalogue of diatoms that inhabit several rivers and streams of Salamanca. This catalogue is made up of 210 taxa which belong to 164 species. We think that 14 of them are new for Spanish alga flora. We also present some tables where several physical-chemical factors of the water

Key words. Diatoms, alga, phytobenthos, fresh waters, Salamanca, Spain.

\section{INTRODUCCIÓN}

Las diatomeas componen normalmente la mayor fracción algal de las aguas corrientes, por lo que su estudio reviste un gran interés. Además, resultan instrumentos valiosos a la hora de averiguar el grado de contaminación de los cursos de agua (Descy, 1979; Almeida Rino y Gil, 1987; Sabater et al., 1987).
Este trabajo se centra en la identificación de especies y en la distribución de las mismas.

\section{MATERIAL Y MÉTODOS}

Para llevar a cabo este estudio dispusimos una primera red de 27 estaciones de muestreo que visitamos a finales de noviembre

Este trabajo se encuadra dentro del Proyecto Análisis de la contaminación y regeneración de los cauces fluviales de la provincia de Salamanca (excepción rio Tormes), realizado gracias a una ayuda de la Excma. Diputación de Salamanca. 


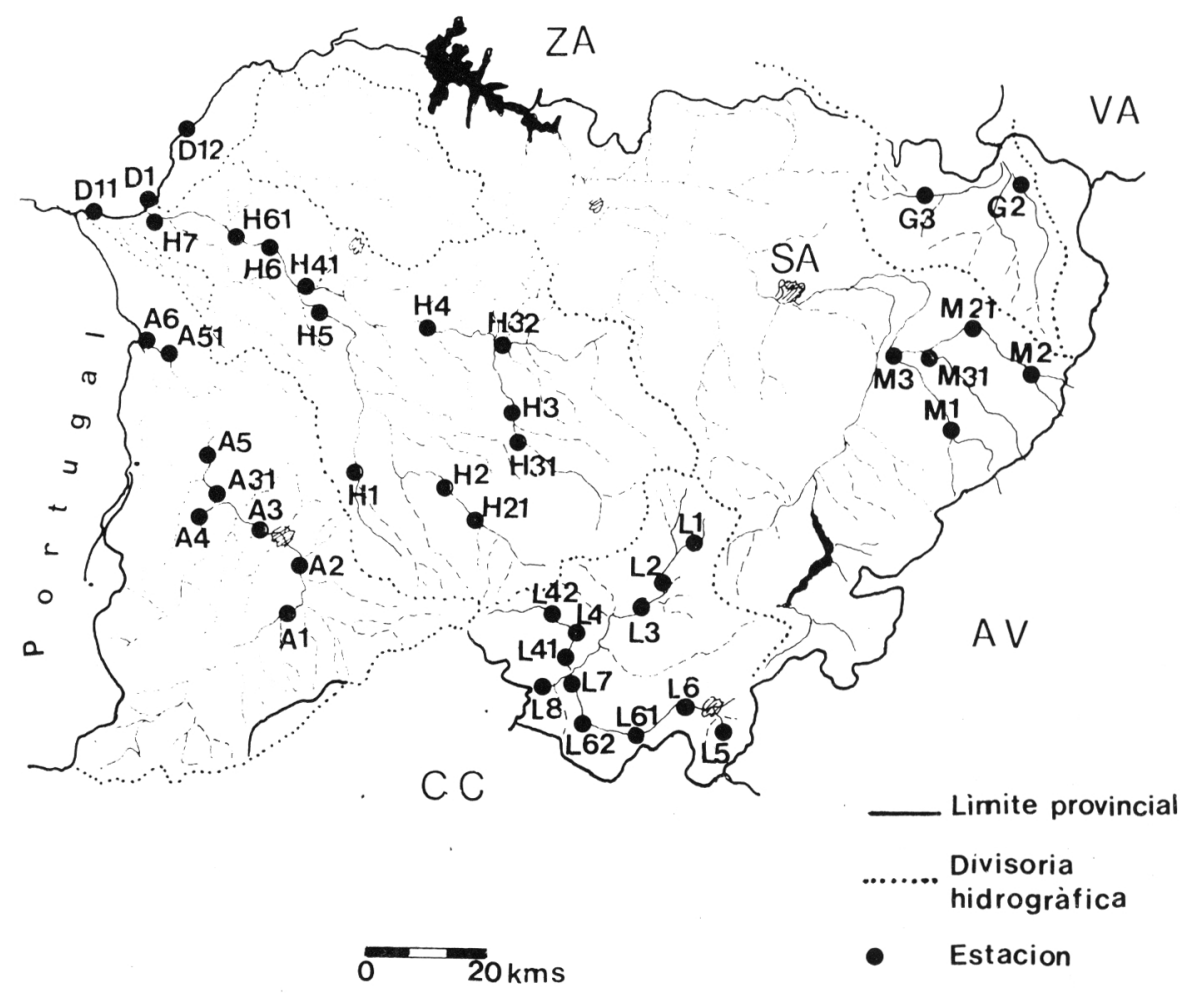

Mapa 1. Situación de las estaciones estudiadas.

de 1987 , en marzo y septiembre de 1988 y, por último, a mediados de febrero de 1989. Acabada la primera visita otoñal, añadimos quince estaciones más.

El nombre de cada punto de recogida se abrevia con dos siglas cuando ha sido visitado en cuatro ocasiones y con tres siglas cuando pertenece a la segunda red (tab. 1).

Las tomas de agua para los análisis químicos fueron efectuadas a la vez que las nuestras por varios miembros del Departamento de Química Analítica de la Universidad de Salamanca.

En cada estación se recogieron muestras de sustratos diversos (briófitos, fanerófitos enraizados, piedras del fondo, etc.), que fueron incluidas en botes opacos para su transporte. De vuelta al laboratorio, las recolecciones se fijaron con formaldehído al $4 \%$ y se conservaron en frío hasta su preparación y montaje.

La obtención de frústulos limpios se llevó a cabo siguiendo el método tradicional del ácido nítrico. Tras repetidos lavados con agua destilada, las muestras se montaron en bálsamo de Canadá. De cada toma, 153 en total, se prepararon cuatro placas para su observación a 1000 aumentos.

Para las determinaciones se utilizaron las claves de Hustedt (1930), Bourrely (1968), Germain (1981) y Patrick \& Reimer (1975). A cada uno de los táxones determinados en las preparaciones, se le asignó una frecuencia siguiendo el criterio de Margalef (1948:258).

Los factores físico-químicos del medio estudiados han sido $\mathrm{pH}$, temperatura, DQO, amonio-amoniaco, nitrito, nitrato, 
Cuenca del río Agueda (Duero). A1.- Río Agueda, antes de la confluencia con el río Agadón, 740 m., 29TQE132876. A2.- Río Agueda, «dehesa de Cuadrados», 740 m, 29TQE132906. A3.- Río Agueda, Ciudad Rodrigo, 680 m, 29TQE082970. A31.- Río Agueda, confluencia con el río Azaba, 590 m., 29TPF982006. A4.- Río Azaba, «Granja de Marialba», 590 m., 29TPE982988. A5.- Río Agueda , entre Martillán y Castillejo de Martín Viejo, 580 m., 29TPF980066. A51.- Río Agueda, Puerto Seguro, 390 m , 29TPF908222. A6.- Río Agueda, «muelle de Vega de Terrón», 290 m., 29TPF742346. Cuenca del río Huebra (Duero). H1.- Río Gavilanes, Sancti-Spiritus, 810 m., 29TQF190102. H2.- Río Yeltes, Aldehuela de Yeltes, 880 m., 29TQF340048. H21.- Río Yeltes, Puebla de Yeltes, 890 m., 29 TQF388010. H3.- Río Huebra, San Muñoz, 780 m., 29TQF426192. H31.- Río Huebra, Buenabarba, 890 m., 29TQF428150. H32.- Río Huebra, entre Aldeadávila de Revilla y Garcibuey, 780 m., 29TQF388290. H4.- Río Huebra, El Cubo de Don Sancho, 740 m., 29TQF260314. H41.- Río Huebra, confluencia con el río Yeltes, 740 m., 29TQF086346. H5.- Río Yeltes, entre Villavieja y Yecla de Yeltes, 745 m., 29TQF126316. H6.- Río Huebra, cercanías de Cerralbo, 730 m., 29TQF052402. H61.- Río Huebra, entre Bermellar y Saldeana, 590 m., 29 TPF996416. H7.- Río Huebra, presa de Saucelle, 280 m., 29TPF846450. Cuenca del río Alagón (Tajo). L1.- Río Alagón, Monleón, 900 m., 30TTK606972. L2.- Río Alagón, San Esteban de la Sierra, 600 m., 30TTK550886. L3.- Río Alagón, Los puentes del Alagón, 500 m., 30TTK506870. L4.- Río Francia, Miranda del Castañar, 600 m., 29TQE538860. L41.- Río Francia, confluencia con el Alagón, 460 m., 29TQE536808. L42.- Río Francia, San Martín del Castañar, 700 m., 29TQE490894. L5.- Río Cuerpo de Hombre, entre Candelario y Bejar, 980 m., 30 TTK664724. L6.- Río Cuerpo de Hombre, Bejar, 850 m., 30 TTK626750. L61.- Río Cuerpo de Hombre, Montemayor del Río, 700 m., 30TTK550706. L62.Río Cuerpo de Hombre, Valdelageve, 600 m., 30TTK593250 L7.- Río Cuerpo de Hombre, Colmenar de Montemayor, 530 m., 30TTK564730. L8.- Río Alagón, Sotoserrano, 390 m., 29TQE522786. Cuenca del río Almar (Duero). M1.- Río Agudín, Gajates, 900 m., 30TUL006164. M2.- Río Almar, proximidades de Bóveda del río Almar, 900 m., 30TUL126272. M21.- Río Almar, Ventosa del río Almar, 880, 30TUL028332. M3.- Río Gamo, entre La Granja y Azud, 860 m., 30TTL928282. M31.- Río Margañan, Peñarandilla, 860 m., 30TTL988286. Cuenca del río Guareña (Duero). G2.- Río Mazores, cercanías de Tarazona de Guareña, 790 m.,. 30TUL068596. G3.- Río Guareña, Espino de la Orbada, 790 m., 30TTL960536. Cuenca del río Duero. D1.- Río Duero, confluencia con el río Huebra-Yeltes, 200 m., 29TPF850470. D11.- Río Duero, confluencia con el río Agueda, 180 m., 29TPF744446. D12.- Río Duero, proximidades del Salto de Aldeadávila, 200 m., 29TPF926624.

Tabla 1.- Localidades estudiadas.

conductividad, fósforo reactivo soluble, cloruro y sulfato (tab. 2). Los valores de $\mathrm{pH}$ se han medido potenciométricamente con un electrodo selectivo, los de temperatura con un termómetro, los de DQO siguiendo el método del permanganato, los de amonio-amoniaco por destilación del amonio y determinación espectrofotométrica con el reactivo de Nessler, el nitrito por determinación espectrofotométrica con el reactivo de Zambelli, el nitrato por determinación espectrofotométrica con el reactivo brucinaácido sulfanílico, la conductividad con un conductímetro, el fosfato por determinación espectrofotométrica con molibdato amónico y ácido ascórbico, el cloruro mediante el método de Mohr y, finalmente, el sulfato por determi- nación turbidimétrica con sulfato de bario.

Se añaden 10 láminas en las que aparecen fotografiadas la mayoría de los táxones. Con muy pocas excepciones, las tomas fotográficas se realizaron a 1000 aumentos.

El depósito de muestras que avalan este trabajo se encuentra en el Departamento de Biología Vegetal (Unidad de Biología General) de la Universidad de Salamanca.

\section{RESULTADOS}

En este primer estudio sobre la flora diatomológica de los ríos salmantinos hemos reconocido 196 taxa pertenecientes a 160 especies.

En cada especie se mencionan las loca- 
lidades de recogida y, cuando existe, su variabilidad. Además, se señalan el número de observaciones (n) y el sumatorio de las frecuencias $\left(\sum \mathrm{H}\right)$ como valores orientativos de la frecuencia y abundancia de cada una de ellas, respectivamente. Tampoco hemos desaprovechado la oportunidad de destacar otros aspectos que nos han parecido importantes.

\section{Achnanthes bioreti Germain}

$$
(\mathrm{n}=6, \Sigma \mathrm{H}=7)
$$

Localidades: A $(1,3,4)$, L $(41,5,6)$.

Achnanthes clevei Grun.

$\left(\mathrm{n}=16, \sum \mathrm{H}=20\right)$. (Fig. $\left.2: 21-22\right)$

Localidades: A $(3,31,5), \mathrm{H}(5,6,61,7), \mathrm{L}(2$, 61, 7), M (3), G (2), D (1, 11).

\section{Achnanthes coarctata Bréb.}

$(n=3, \Sigma H=4)$. (Fig. 2: 12-14)

Algunos ejemplares no muestran constricción mediana alguna (figs. 13-14). Localidades: H (6), G (2).

Achnanthes exigua Grun. var. heterovalvata Krasske

(n=31, $\Sigma \mathrm{H}=46)$. (Fig. 2: 24)

Localidades: A, H (32, 41, 5, 6, 61, 7), L (2, 61, 7), M (1, 3, 31).

\section{Achnanthes hungarica Grun.}

$(\mathrm{n}=25, \Sigma \mathrm{H}=45)$. (Fig. 2: 15-16)

Localidades: A $(4,5), \mathrm{H}(1,21,3,4,41,5,6$, 61), L (2, 8), M (1, 2, 21), G.

\section{Achnanthes lanceolata Bréb}

$(\mathrm{n}=129, \Sigma \mathrm{H}=313$ ). (Fig. 2: 17-19)

Las variedades rostrata Hust. (fig. 18) y elliptica $\mathrm{Cl}$. (fig. 19) así como numerosas formas de tránsito, suelen acompañar al tipo de la especie.

Localidades: Todas las estudiadas.

\section{Achnanthes minutissima gr.}

(Fig. 2: 20)

Lo anotamos así porque se trata de un conjunto frecuente en nuestras estaciones y que ha sido difícil tratar a mil aumentos.

Localidades: Excepto M3.

Achnanthes peregalli Brun. \& Heribaudi $(n=4, \Sigma H=4)$. (Fig. 2: 23)
Se presenta en aguas muy pobres en sales y oxigenadas.

Medidas: Valvas de $15 \mu \mathrm{m}$ de longitud y $7 \mu \mathrm{m}$ de anchura.

Localidades: A (1, 3, 5), H (41).

Amphipleura pellucida Kütz.

(n=13, $\mathrm{H}=20$ ). (Fig. 3: 20 )

Localidades: H (3, 31, 32, 4, 41, 5, 7), L (3), G (2).

Amphora ovalis Kütz.

( $\mathrm{n}=45, \Sigma \mathrm{H}=55)$. (Fig. 7: 1-2)

Localidades: A $(1,3,4,5,51), \mathrm{H}(2,3,31,32$, $41,5,6,61,7), \mathrm{L}(1,2,3,62,7), \mathrm{M}$ (excepto 3), G , D $(1,11)$.

Amphora pediculus Kütz.

( $\mathrm{n}=29, \sum \mathrm{H}=45$ ). (Fig. 7: 3)

Localidades: A $(3,31,4,5,51,6), \mathrm{H}$ (excepto

1 y 21$), \mathrm{L}(2,3), \mathrm{M}(1,3,31), \mathrm{G}, \mathrm{D}(1,11)$.

Amphora veneta Kütz.

( $\mathrm{n}=45, \Sigma \mathrm{H}=49$ ). (Fig. 7: 4-5)

Localidades: A $(31,4,5,6), \mathrm{H}(2,32,4,41,5$,

6, 61, 7), L (1, 2, 61), M (excepto 3), G , D (11).

Anomoeoneis sphaerophora (Kütz.) Pfitzer

$(\mathrm{n}=3, \Sigma \mathrm{H}=3)$. (Fig. 3: 6)

Localidades: H (61), M (1), G.

Asterionella formosa Hassal

$(\mathrm{n}=3, \Sigma \mathrm{H}=3$ )

Localidades: A $(2,3,51,6), \mathrm{H}(41,7), \mathrm{D}(1$, 11).

Caloneis alpestris (Grun.) $\mathrm{Cl}$.

$(\mathrm{n}=1, \Sigma \mathrm{H}=2)$

Localidad: M (31).

Caloneis amphisbaena (Bory) $\mathrm{Cl}$.

(n=1, $\mathrm{H}=1$ ). (Fig. 3: 16)

Localidad: $\mathrm{G}(2)$.

Caloneis bacillum (Grun.) Meresch. var. fontinalis Grun.

$(\mathrm{n}=9, \Sigma \mathrm{H}=9$ ). (Fig. 3: 19)

G (2).

Localidades: A (4), H (1, 31, 32, 5), L (4, 6),

Caloneis ventricosa (Ehr.) Meister $(\mathrm{n}=31, \Sigma \mathrm{H}=32$ ). (Fig. 3: 17-18) 


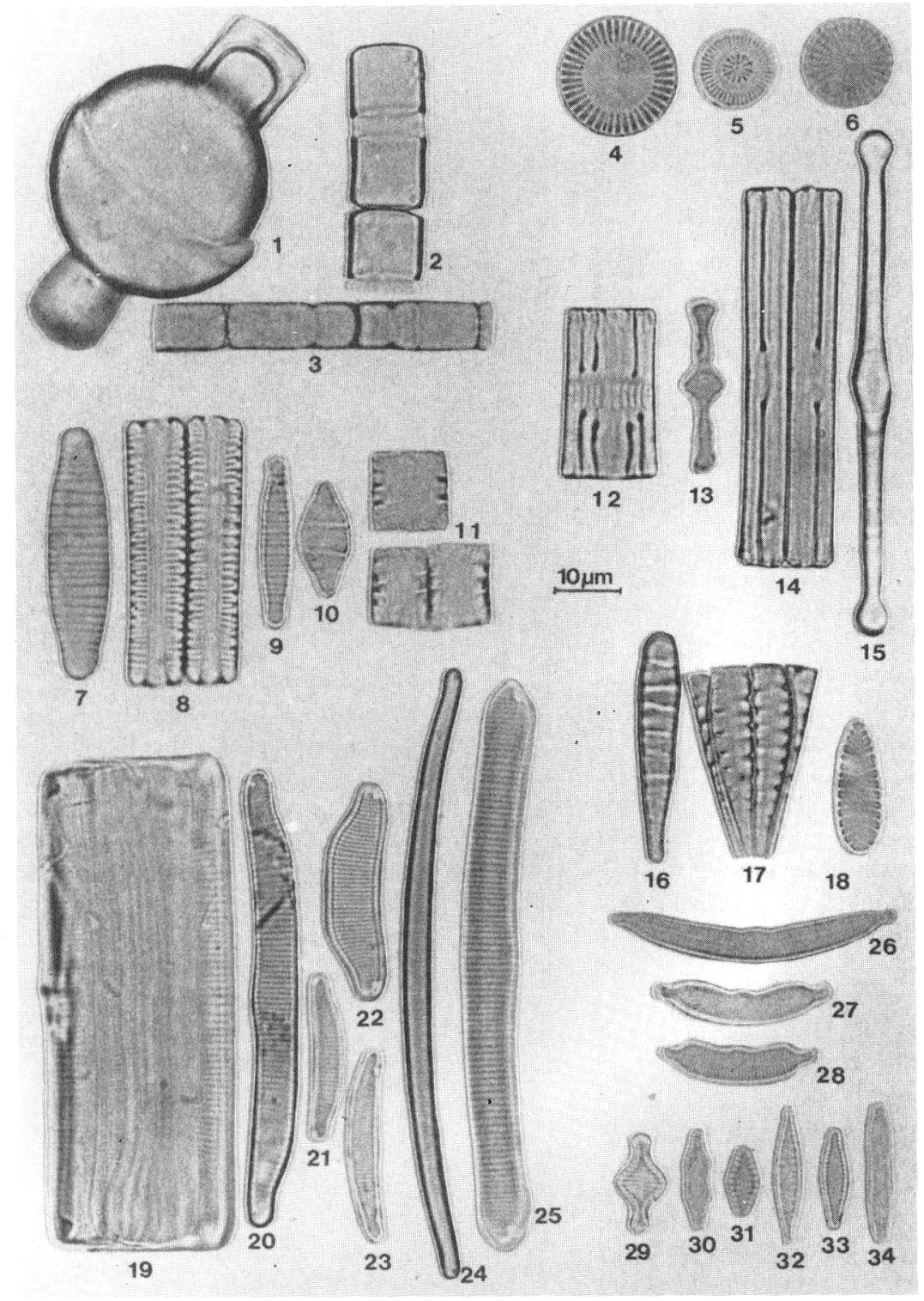

Figura 1. 1. Filamento de Melosira varians con auxospora.- 2. M. varians. - 3 M. ambigua. - 4 Cyclotella meneghiniana. - 5. C. stelligera. - 6. Stephanódiscus astraea. - 7. Diatoma vulgare. - 8. D. vulgare, colonia -9. D. elongatum var. tenuis. - 10. D. hiemale var. mesodon. - 11. D. hiemale var. mesodon, vista conectiva y colonia de dos indiviudos. - 12 Tabellaria flocculosa, colonia - 13. T. flocculosa. - 14. T. fenestrata, colonia. - 15. T. fenestrata. - 16. Meridion circulare. - 17. M. circulare, colonia pseudoflabeliforme. - 18. M. circulare var. constricta. - 19. Eunotia pectinalis, colonia. - 20. E. pectinalis var. ventralis. - 21. E. pectinalis var. rostrata. - 22. E. diodon. - 23. E. veneris. - 24. E. lunaris. - 25. E. formica. - 26. Ceratoneis arcus. - 27. C. arcus, forma de paso hacia la variedad amphioxys.- 28. C. arcus var, amphioxys. - 29. Fragilaria construens. - 30. F. construens var. binodis. - 31. F. pinnata var. lancettula. - 32. F. pinnata. - 33. F. brevistriata. - 34. F. capucina var. mesolepta. 
La variedad truncatula (Grun.) Meist. (fig. 18), fue recogida exclusivamente en $\mathrm{H} 4$.

Localidades: A $(3,31,5,51), \mathrm{H}(1,21,31,32$, $4,41,5,6), \mathrm{L}(1,2,3,8), \mathrm{M}, \mathrm{G}(2), \mathrm{D}(1,11)$.

Ceratoneis arcus (Ehr.) Kütz.

( $\mathrm{n}=65, \Sigma \mathrm{H}=180$ ). (Fig. 1: 26-28)

No son raras las formas de paso hacia la rechoncha variedad amphioxys Rabh. (fig. 28).

Localidades: A (excepto 4), H (2, 21, 3, 32, 5, 61), L (excepto 1).

Cocconeis pediculus Ehr.

$(\mathrm{n}=32, \Sigma \mathrm{H}=59)$. (Fig. 2: 10-11)

Localidades: A $(3,5,51,6), \mathrm{H}(2,3,31,32$, 41, 6 61, 7), L (2), M (excepto 31), D (1).

Cocconeis placentula Ehr.

$(\mathrm{n}=140, \Sigma \mathrm{H}=400)$. (Fig. 2: 8-9)

Localidades: Todas las estudiadas.

Coscinodiscus lacustris Grun.

$\left(\mathrm{n}=1, \sum \mathrm{H}=1\right)$

Localidad: L (2).

Cyclotella meneghiniana Kütz.

$(\mathrm{n}=119, \Sigma \mathrm{H}=257)$. (Fig. 1:4)

Localidades: Excepto L (41, 42, 62).

Cyclotella stelligera Cl. \& Grun.

$(\mathrm{n}=9, \Sigma \mathrm{H}=14)$. (Fig. 1:5)

Localidades: A $(2,3,31,5,6)$.

Cymatopleura elliptica (Bréb.) W. Sm.

( $\mathrm{n}=11, \sum \mathrm{H}=11$ ). (Fig. 8: 17)

Localidades: H (3, 4, 41, 6, 7), M (3), G (2).

Cymatopleura solea (Bréb.) W. Sm.

$(\mathrm{n}=49, \Sigma \mathrm{H}=68)$. (Fig. 8: 18)

Localidades: A $(3,31,51,6), \mathrm{H}(3,32,4,41$,

$5,6,61,7), \mathrm{L}(2,3), \mathrm{M}, \mathrm{G}, \mathrm{D}(1)$.

Cymbella aspera (Ehr.) $\mathrm{Cl}$.

$\left(\mathrm{n}=3, \sum \mathrm{H}=6\right)$. (Fig. 6: 2-3)

Localidades: H $(3,6), \mathrm{G}(2)$.

Cymbella caespitosa (Kütz.) Brun.

( $\mathrm{n}=13, \Sigma \mathrm{H}=24)$. (Fig. 6: 10)

Localidades: H (3, 31, 41, 5, 6, 61, 7), L (2),

M (1), D (1).
Cymbella cesatii (Rabh.) Grun.

$(\mathrm{n}=1, \Sigma \mathrm{H}=1)$

Localidad: L (2).

Cymbella cistula (Hemprich) Grun.

$(\mathrm{n}=34, \Sigma \mathrm{H}=58)$. (Fig. 6: 4-5)

Localidades: A $(3,4,51,6), \mathrm{H}(1,3,31,32,4$,

$41,5,6,61,7)$, L (1, 2, 3), M (1, 2).

Cymbella heteropleura Ehr.

$(\mathrm{n}=16, \Sigma \mathrm{H}=18)$. (Fig. 6: 16)

Localidades: A (1, 3), H (2, 21, 31, 6), L (3, 4,

$42,62,8), \mathrm{M}(1)$.

Cymbella lanceolata (Ehr.) V. Heurck

$(\mathrm{n}=35, \Sigma \mathrm{H}=49)$. (Fig. 6: 1)

Localidades: A $(4,5,51), \mathrm{H}(3,32,4,41,5$,

$6,61,7), \mathrm{L}(1,2,8), \mathrm{M}(21,3), \mathrm{G}, \mathrm{D}(1,12)$

Cymbella leptoceros (Ehr.) Grun.

$(\mathrm{n}=1, \Sigma \mathrm{H}=1)$. (Fig. 6: 15)

Localidades: $\mathrm{H}$ (3)

Cymbella minuta Hilse ex Rabh.

$(\mathrm{n}=136, \Sigma \mathrm{H}=305)$. (Fig. $6: 12-14)$

Localidades: Todas las estudiadas.

Cymbella parva (W. Sm.) Cl.

$(n=4, \Sigma H=18)$. (Fig. 6: 6)

Localidades: $\mathrm{H}(3,4,6)$.

Cymbella prostrata (Berkeley) $\mathrm{Cl}$.

$(\mathrm{n}=10, \Sigma \mathrm{H}=18)$. (Fig. 6: 9)

Localidades: A (5), M (3), G (2), D.

Cymbella sinuata Greg. fma. ovata Hust.

$(\mathrm{n}=23, \Sigma \mathrm{H}=26)$. (Fig. 6:17)

Localidades: A, H (2, 41, 5, 6, 7), L (2, 3, 4, 41), M (31), G (2).

Cymbella tumida (Bréb.) V. Heurck

$(\mathrm{n}=67, \Sigma \mathrm{H}=113$ ). (Fig. 6: 7-8)

Aunque es excepcional, a veces presenta dos estigmas (fig. 7).

Localidades: A (excepto 1), H, L (1, 2, 3, 7, 8), M (3), G (2 ), D (1).

Cymbella turgida (Greg.) $\mathrm{Cl}$.

$(\mathrm{n}=10, \Sigma \mathrm{H}=11)$. (Fig. 6: 11)

Localidades: A $(1,3,31), \mathrm{H}(2,4,6,61,7), \mathrm{G}$ 


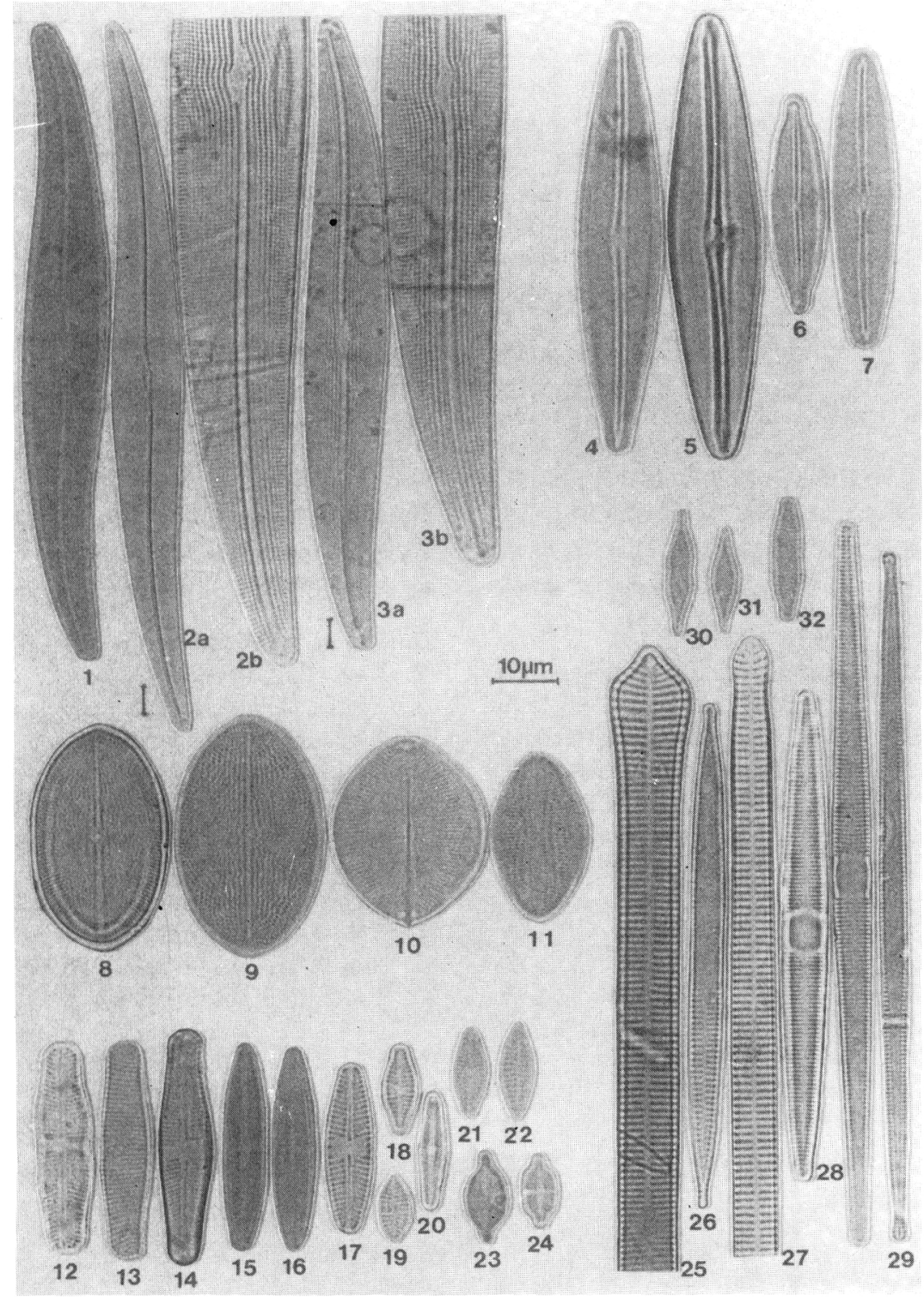

Figura 2. 1.Gyrosigma acuminatum. - 2a. G. attenuatum (x 400). - 2b.- G. attenuatum, detalle del área central. 3a. G. attenuatum (x 400) 3b. G. attenuatum, detalle de la estriación. - 4.Frustulia rhomboides var. saxonica. - 5. F. rhomboides var viridula. - 6. F. rhomboides var. saxonica fma capitata. - 7. F. vulgaris. - 8. Cocconeis placentula, hipovalva. - 9. C. placentula, epivalva. - 10. C. pediculus, hipovalva. - 11. C. pediculus, epivalva. - 12. Achnanthes coarctata, ejemplar con una ligera constricción mediana. -13. A coarctata, epivalva. - 14. A. coarctata, hipovalva. - 15. A. hungarica, hipovalva. - 16. A. hungarica, epivalva. - 17. A. lanceolata. - 18. A. lanceolata var. rostrata. - 19. A. lanceolata var. elliptica. - 20. A. minutissima. - 21. A. clevei, hipovalva. - 22. A. clevei, epivalva. - 23. A. peregalli. - 24. A. exigua var. heterovalvata. - 25. Synedra capitata. - 26. S. ulna var. biceps. - 27. S. ulna. - 28. S. pulchella. - 29. S. tabulata. - 30. S. parasitica. - 31. S. parasitica var. subconstricta. - 32. S. vaucheriae. 
Denticula tenuis Kütz. var. crassula Naegeli

$(\mathrm{n}=1, \Sigma \mathrm{H}=1)$

Localidades: $\mathrm{G}(2)$

Diatoma elongatum (Lyngb.) Ag.

$\left(\mathrm{n}=8, \sum \mathrm{H}=10\right)$. (Fig. 1: 9)

Aparecen formas intermedias entre el tipo de la especie y la variedad tenuis (Ag.) Kütz., siendo la más frecuente ésta última.

Localidades: A $(31,6), \mathrm{H}(2,41,5), \mathrm{D}(11)$.

D. hiemale (Lyng.) Heiberg var. mesodon (Ehr.) Grun.

$(\mathrm{n}=31, \Sigma \mathrm{H}=57)$. (Fig. 1: 10-11)

Localidades: A $(1,2), \mathrm{H}(2,21,3), \mathrm{L}(3,4,41$, $42,5,6,61,62,7)$.

\section{Diatoma vulgare Bory}

( $\mathrm{n}=53, \Sigma \mathrm{H}=147$ ). (Fig. 1: 7-8)

En poblaciones muy numerosas suelen aparecer la var. linearis Grun. y, más raramente, la var. ovalis (Fricke) Hust.; tampoco son infrecuentes entonces los diseños valvares extraordinarios.

Localidades: A $(2,51,6), \mathrm{H}(3,32,4,41,5,6$, $61,7), \mathrm{L}(2,3,8), \mathrm{M}(3), \mathrm{G}(2), \mathrm{D}$

Diploneis ovalis (Hilse) $\mathrm{Cl}$.

$\left(\mathrm{n}=22, \sum \mathrm{H}=26\right)$. (Fig. 3: 8-9)

La variedad oblongella (Naegeli) $\mathrm{Cl}$. (fig. 9) se recogió en las aguas salobreñas del río Mazores.

Localidades: H (3, 31 , 41, 5, 6, 7), L (3, 4), M (3), G, D (1).

Diploneis puella (Schumann) $\mathrm{Cl}$. $\left(\mathrm{n}=18, \sum \mathrm{H}=10\right)$. (Fig. 3: 7)

Localidades: H (3, 6, 61, 7), L (3), M (21), G.

Epithemia sorex Kütz.

( $\mathrm{n}=40, \Sigma \mathrm{H}=70$ ). (Fig. 9: 6)

Localidades: A (4), H (3, 31, 32, 4, 41, 5, 6, 61, 7), L (1, 2), M (31), G (2), D (1, 12).

Epithemia turgida (Ehr.) Kütz. $(\mathrm{n}=55, \Sigma \mathrm{H}=75)$. (Fig. 9: 3-5)

Localidades: A $(2,31,4,5,51,6), \mathrm{H}$ (excepto 1 y 2), L (1, 2, 3, 8), M (1, 2, 31), G (2), D (1, 12).

Epithemia zebra (Ehr.) Kütz.

( $\mathrm{n}=27, \Sigma \mathrm{H}=30$ ). (Fig. 9: 1-2)

Las variedades saxonica (Kütz.) Grun. (fig.
1) y porcellus (Kütz.) Grun. son las más comunes. Localidades: A (4, 5, 51, 6), H (31, 32, 4, 41, $5,6,61,7), \mathrm{L}(1,2,3), \mathrm{M}(21), \mathrm{G}$.

Eunotia lunaris (Ehr.) Grun.

$(\mathrm{n}=10, \Sigma \mathrm{H}=13)$. (Fig. 1:24)

Localidades: A (1), H (2, 21, 5, 6), L (1, 2, 8), M (21, G (2).

Eunotia diodon Ehr. ( $\mathrm{n}=12, \Sigma \mathrm{H}=15$ ). (Fig. 1: 22)

Localidades: A (1, 2), H (1, 2, 21), L (1, 4, 5, $6)$.

Eunotia formica Ehr.

$(\mathrm{n}=2, \Sigma \mathrm{H}=2)$. (Fig. 1: 25)

Localidades: A (3), H (6).

Eunotia pectinalis (Kütz.) Rabh.

( $\mathrm{n}=62, \Sigma \mathrm{H}=127)$. (Fig. 1: 19-21)

No es fácil la distinción de variedades. La figura 21 muestra un ejemplar con características de rostrata Germain y undulata (Ralfs) Rabh.; la siguiente, se asemeja a la fma. impressa Ehr. de la var. minor (Kütz.) Rabh., pero cuenta con un número mayor de estrías ( 20 cada $10 \mu \mathrm{m}$ ); menos corrientes y conflictivas son las variedades ventralis (Ehr.) Hust.(fig. 20) y pectinalis.

Localidades: A (1, 2, 3, 31, 5, 6), H (1, 2, 21 , $3,31,41,5,6)$, L, M (3), G (2).

Eunotia robusta Ralfs

$(\mathrm{n}=1, \Sigma \mathrm{H}=1)$

Localidad: A (1)

Eunotia veneris (Kütz.) O. Müller

$(\mathrm{n}=5, \Sigma \mathrm{H}=5)$. (Fig. 1:23)

Localidades: A $(1,3,31,6)$.

Fragilaria brevistriata Grun.

$(\mathrm{n}=25, \Sigma \mathrm{H}=37)$. (Fig. 1: 33)

Localidades: A $(1,3,31,4,5,6), \mathrm{H}(32,4,41$,

$6,61,7), \mathrm{L}(2), \mathrm{M}(3), \mathrm{G}(2)$.

Fragilaria capucina Desmazieres

$(\mathrm{n}=51, \Sigma \mathrm{H}=130)$. (Fig. 1: 34)

Fotografiamos la frecuente y abundante variedad mesolepta (Rabh.) Grun.

Localidades: A $(2,3,31,4,5,6), \mathrm{H}(3,31,32$, 4, 41, 5, 6, 61, 7), L (1, 2, 7, 8), M (1, 2, 21, 3), G. 


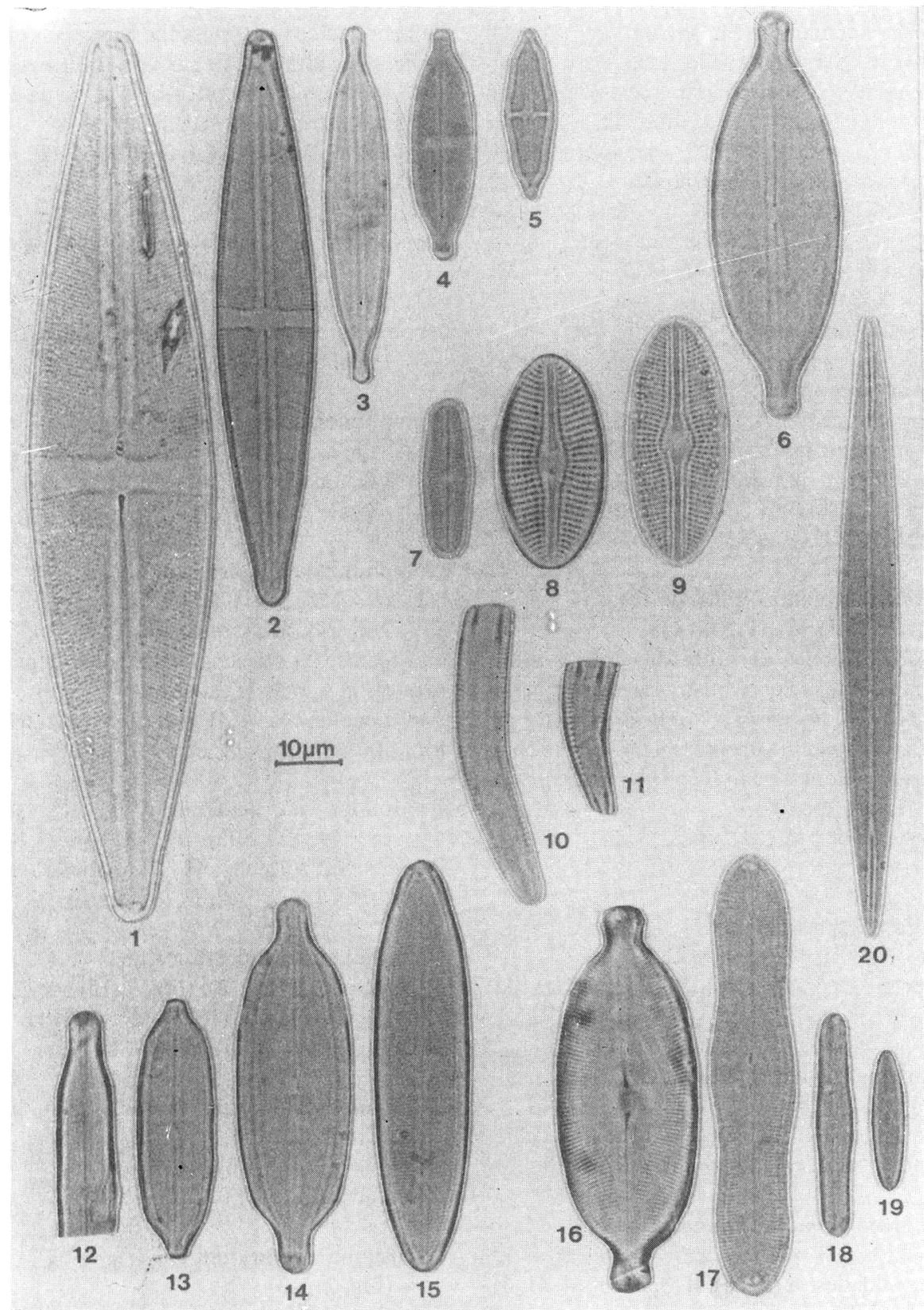

Figura 3. 1. Stauroneis phenicenteron. - 2. S. phenicenteron fma. gracilis. - 3. S. anceps fma. gracilis. - 4. S. anceps. - 5. S. smithii. - 6. Anomoeoneis sphaerophora. - 7. Diploneis puella. - 8. D. ovalis. - 9. D. ovalis var. oblongella. - 10-11. Rhoicosphenia curvata. - 12. Neidiun affine var. amphyrhynchus fma . undulata. - 13. N. dubium. - 14. N. affine-15. N. iridis. - 16. Caloneis amphisbaena. - 17. C. ventricosa. - 18. C. ventricosa var. truncattula. - 19. C. bacillum var. fontinalis. - 20. Amphipleura pellucida. 
Fragilaria construens (Ehr.) Grun.

$\left(\mathrm{n}=76, \sum \mathrm{H}=278\right.$ ). (Fig. 1: 29-30)

$\mathrm{Al}$ menos cuenta con cuatro variedades en nuestras aguas: binodis (Ehr.) Grun. (fig. 30), venter (Ehr.) Grun., construens (fig. 29) y subsalina Hust.

Localidades: A, H (excepto 1 y 2), L (2, 3, 5, 7, 8), M (excepto 2), G (2), D.

\section{Fragilaria crotonensis Kitton}

$(\mathrm{n}=7, \Sigma \mathrm{H}=9)$

Localidades: $\mathrm{H}(4,41,6,61,7), \mathrm{D}(1,2)$

\section{Fragilaria pinnata Ehr.}

$(\mathrm{n}=47, \Sigma \mathrm{H}=97)$. (Fig. 1: 31-32)

Con numerosas formas de paso hacia la variedad lancettula (Schumann) Hust. (fig. 31).

Localidades: A $(3,31,4,5,51,6), \mathrm{H}(3,31,4$, $41,5,6,61,7), \mathrm{L}(1,2,3,8), \mathrm{G}(2), \mathrm{D}(1,11)$.

Frustulia rhomboides (Ehr.) de Toni

$(\mathrm{n}=6, \Sigma \mathrm{H}=6)$. (Fig. 2: 4-6)

Unos pocos ejemplares con bandas longitudinales, e incluso con granulaciones, pertenecientes a la variedad viridula (Bréb.) Cl. (fig. 5), aparecieron esporádicamente; menos raras son la var. saxonica (Rabh.) Toni (fig. 4) y su fma. capitata Mayer (fig. 6). (31).

Localidades: A (51), H (5), L (4, 5, 61), M

Frustulia vulgaris Thwaites

$(\mathrm{n}=74, \Sigma \mathrm{H}=101)$. (Fig. 2: 7)

Localidades: A (excepto 51), H, L, M (excepto 2$), \mathrm{G}(2), \mathrm{D}(1,11)$.

Gomphoneis clevei (Fricke) M. C. Gil

(Fig. 2: 21-24)

Le hemos dedicado un estudio aparte (Gallego Martín et al, aceptado para su publicación).

Gomphoneis olivacea (Lyngb.) Dawson

$(\mathrm{n}=26, \Sigma \mathrm{H}=49$ ). (Fig. 7: 6)

Localidades: A $(51,6), \mathrm{H}(3,31,32,4,41,6)$, L (3), M , G, D (1).

\section{Gomphonema acuminatum Ehr.}

$(\mathrm{n}=99, \Sigma \mathrm{H}=159)$. (Fig. 7: 7-11)

Las variedades brebissonii (Kütz.) $\mathrm{Cl}$. (fig. 11), coronata (Ehr.) W. Sm. (fig. 8) y elongata W. Sm. (fig. 9) no son frecuentes. La última variedad de las relacionadas, evita las aguas con elevados índices de sulfatos y de sustancias nitrogenadas.

Medidas (var. elongata): Valvas de $82 \mu \mathrm{m} \mathrm{x}$ $15 \mu \mathrm{m} ; 9$ estrías cada $10 \mu \mathrm{m}$.

Localidades: A, H, L (1, 2, 3, 61, 62, 7, 8), M, G, D (1).

Gomphonema angustatum (Kütz.) Rabh. $\left(\mathrm{n}=138, \sum \mathrm{H}=364\right)$. (Fig. 7: 18-19)

Las frecuencias y abundancias mayores son las de la variedad producta (Kütz.) Rabh. (fig. 19).

Localidades: Todas las estudiadas.

Gomphonema augur Ehr.

$(\mathrm{n}=23, \Sigma \mathrm{H}=34)$. (Fig. 7: 12-13)

Localidades: A (excepto 2), H (1, 3, 32, 45, 6), L (3), G (2), D (1).

Gomphonema constrictum Ehr.

( $\left.\mathrm{n}=118, \sum \mathrm{H}=281\right)$. (Fig. $\left.7: 16-17\right)$

La variedad capitata (Ehr.) Cl. (fig. 17), aunque aparece en pequeñas cantidades, acompaña a menudo al tipo de la especie.

Localidades: A, H, L (1, 2, 3, 4, 41, 61, 62, 7, 8), M (excepto 31), G, D.

\section{Gomphonema gracile Ehr.}

$(\mathrm{n}=49, \Sigma \mathrm{H}=93)$. (Fig. 7: 20)

Localidades: A (menos 51), $\mathrm{H}(\operatorname{menos} 32,61$ y 7$)$, $\mathrm{L}(3,4,5,61,62,7,8), \mathrm{M}$ (excepto 3 ), G (2).

\section{Gomphonema intricatum Kütz.}

$(\mathrm{n}=16, \Sigma \mathrm{H}=18)$. (Fig. $7: 15)$

Localidades: A $(1,2,4,51,6), \mathrm{H}(1,21,31$, $41,6), \mathrm{L}(3,62,7), \mathrm{M}(1), \mathrm{G}$.

Gomphonema lanceolatum Ehr. var. insignis (Greg.) $\mathrm{Cl}$.

$\left(\mathrm{n}=2, \sum \mathrm{H}=2\right.$ ). (Fig. 7: 14)

Localidades: H (41), L (8).

\section{Gyrosigma attenuatum gr.}

(Fig. 2: 1-3)

Con el aumento a que hemos trabajado no siempre es sencillo distinguir las valvas de $G$. attenuatum (Kütz) Rabh. (figs. 2-3) de las de $G$. acuminatum (Kütz.) Rabh. (fig.1). Algunos ejemplares de ésta última muestran una talla sorprendentemente grande (hasta $275 \times 27 \mu \mathrm{m}$ ).

Ambas especies se presentan preferentemen- 


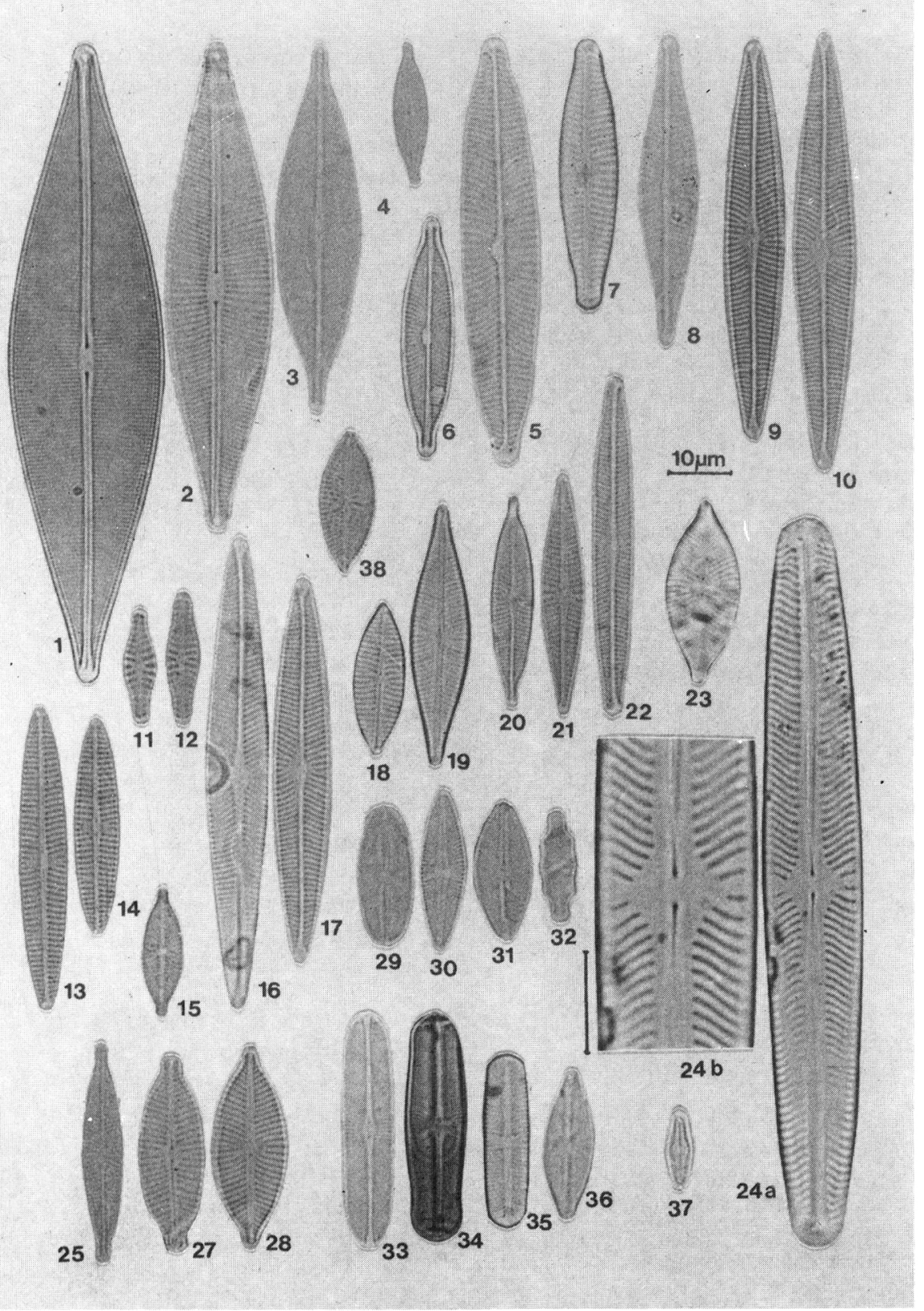

Figura 4. 1. Navicula cuspidata. - 2. Navicula cuspidata var. heribaudi. - 3. N. cuspidata var. ambigua. - 4. N. gregaria. - 5. N. viridula. - 6. N. rostellata. - 7. N. scleviscensis. - 8. N. rhynchocephala - 9, 10 y 17. N. lanceolata. - 11. N. hungarica var. capitata. - 12. N. hungarica.-13-14. N. gracilis. - 15. N. deccusis. - 16 N. radiosa. - 18. N. menisculus. - 19. N. trivialis. - 20. N. capitoradiata. - 21. N. pseudolanceolata. - 22. N. cari var. angusta. - 23. N. clementis. - 24a. N. oblonga. - 24b. $N$. oblonga, detalle de la curvatura del rafe. - 25. $N$. cryptocephala. - 27. $N$. dicephala. - 28. $N$. placentula. - 29. $N$. pygmaea. - 30. $N$. goppertiana. - 31. N. cocconeiformis. - 32. N. pseudonivalis. - 33. N. bacillum. - 34. N. pupula var. capitata. - 35. N. laevissima. - 36. N. nyassensis. - 37. N. perpusilla. - 38. N. pseudotuscula. 
te en las aguas mineralizadas de la cuenca de los ríos Almar y Guareña.

Gyrosigma scalproides (Rabh.) $\mathrm{Cl}$.

$\left(\mathrm{n}=3, \sum \mathrm{H}=3\right)$

Localidades: A (4), H (61).

Hantzschia amphioxys (Ehr.) Grun.

$(\mathrm{n}=59, \Sigma \mathrm{H}=77)$. (Fig. 9: 11-12)

La variedad maior Grun. (fig. 12), fue recogida en la primera estación del río Agueda.

Localidades: A $(1,2,3,31,4,5,6), \mathrm{H}(1,2,3$, $31,4,5,6,7), \mathrm{L}(1,3,4,42,6,61,7,8), \mathrm{M}, \mathrm{G}, \mathrm{D}$ (11).

Melosira ambigua $O$. Müller

$(\mathrm{n}=56, \Sigma \mathrm{H}=93)$. (Fig. 1: 3)

Localidades: A (excepto 1), H (1, 32, 41, 5, 6, 61), L (2, 5, 6, 61, 62, 7, 8), M (3, 31), G (3), D.

\section{Melosira varians Ag.}

$(\mathrm{n}=113, \Sigma \mathrm{H}=311$ ). (Fig. 1: 1-2)

Localidades: Todas las estudiadas

\section{Meridion circulare Ag.}

( $\mathrm{n}=72, \sum \mathrm{H}=111$ ). (Fig. 1: 16-18)

Las variedades circulare y constricta (Ralfs)

V. Heurck (fig. 18) aparecen a menudo mezcladas; no es extraño observar colonias pseudoflabeliformes de cuatro individuos (fig. 17).

Localidades: A (1, 2, 3, 31, 4, 6), H (1, 2, 21, $3,31,4,41,5,6,7)$, L , M (1, 2, 31), G, D (11).

\section{Navicula bacillum Ehr.}

( $\mathrm{n}=28, \Sigma \mathrm{H}=41$ ). (Fig. 4: 33)

Localidades: A $(3,31,4,5,51,6), \mathrm{H}(3,41,6$,

7), L (2, 3, 8), M (21, 3, 31), G (2), D (11).

\section{Navicula capitoradiata Germain}

$(\mathrm{n}=25, \Sigma \mathrm{H}=57$ ). (Fig. 4: 20)

Localidades: A (excepto 2), H (1, 32, 4, 41, 5, $6,61,7), \mathrm{L}(2), \mathrm{M}(1,31), \mathrm{G}(2), \mathrm{D}(11)$.

Navicula cari Ehr. var. angusta Grun.

( $\left.\mathrm{n}=7, \sum \mathrm{H}=11\right)$. (Fig. 4: 22)

Localidades: A (1, 2, 3), H (21, 61), L (5).

Navicula cincta (Ehr.) Kütz.

$(\mathrm{n}=1, \Sigma \mathrm{H}=1)$

Localidades: $\mathrm{H}(1,3), \mathrm{G}(2)$.

Navicula clementis Grun.

$(\mathrm{n}=6, \Sigma \mathrm{H}=6)$. (Fig. 4: 23)

Localidades: A (4), H (6), L (4).
Navicula cocconeiformis Greg.

$(\mathrm{n}=1, \Sigma \mathrm{H}=1)$. (Fig. 4: 31)

Localidades: L (2).

Navicula cryptocephala Kütz.

( $\mathrm{n}=70, \Sigma \mathrm{H}=197)$. (Fig. 4: 25-26)

Localidades: Excepto G3, D12.

Navicula cuspidata Kütz.

$(\mathrm{n}=49, \Sigma \mathrm{H}=73$ ). (Fig. $4: 1-3$ )

En el río Mazores sorprendimos un frústulo de la variedad heribaudi Peregallo (fig. 2); el tipo, del que se ven formas craticulares, y la var. ambigua (Ehr.) Cl. (fig. 3) son bastante comunes.

Localidades: A $(1,3,31,4,5,51,6), \mathrm{H}(1,2$, $3,31,4,41,5,6)$, L (1, 2, 3, 5, 6, 61, 8), M, G.

\section{Navicula deccusis Oestrup}

( $\mathrm{n}=14, \Sigma \mathrm{H}=21$ ). (Fig. 4: 15)

No siempre presenta estigma.

Localidades: H $(6,61), \mathrm{L}(2,7), \mathrm{M}$ (excepto

2), G (2), D (11).

Navicula dicephala (Ehr.) W. Sm.

$(\mathrm{n}=12, \Sigma \mathrm{H}=18)$. (Fig. $4: 27)$

Localidades: A (4), H $(5,6), \mathrm{L}(3,62), \mathrm{M}$

(excepto 3), G (2).

Navicula goppertiana (Bleisch) Grun.

$(\mathrm{n}=31, \Sigma \mathrm{H}=70$ ). (Fig. 4: 30)

Localidades: A $(3,31,5,51,6), \mathrm{H}(4,7), \mathrm{L}$ $(62,7,8), M(3,31), \mathrm{D}$.

Navicula gracilis Ehr.

$(\mathrm{n}=45, \Sigma \mathrm{H}=98)$. (Fig. 4: 13-14)

Localidades: A $(1,2,3,6), \mathrm{H}(2,3,4,41,6,61$,

7), L (2, 3, 41, 5), M (1, 2, 3), G (2), D.

Navicula gregaria Donkin

$(\mathrm{n}=12, \Sigma \mathrm{H}=21)$. (Fig. 4: 4)

Localidades: H $(1,3,5,61)$, L (8), M (3, 31),

G (2), D (1, 11).

Navicula hungarica Grun.

( $\mathrm{n}=43, \Sigma \mathrm{H}=52$ ). (Fig. 4: 11-12)

La variedad capitata (Ehr.)Cl. (fig. 11), está más difundida que el tipo; también existen formas intermedias entre ambas. Frecuentan las aguas turbias y de elevado residuo salino.

Medidas: $42-44 \mu \mathrm{m}$ de longitud x 10-11 $\mu \mathrm{m}$ 


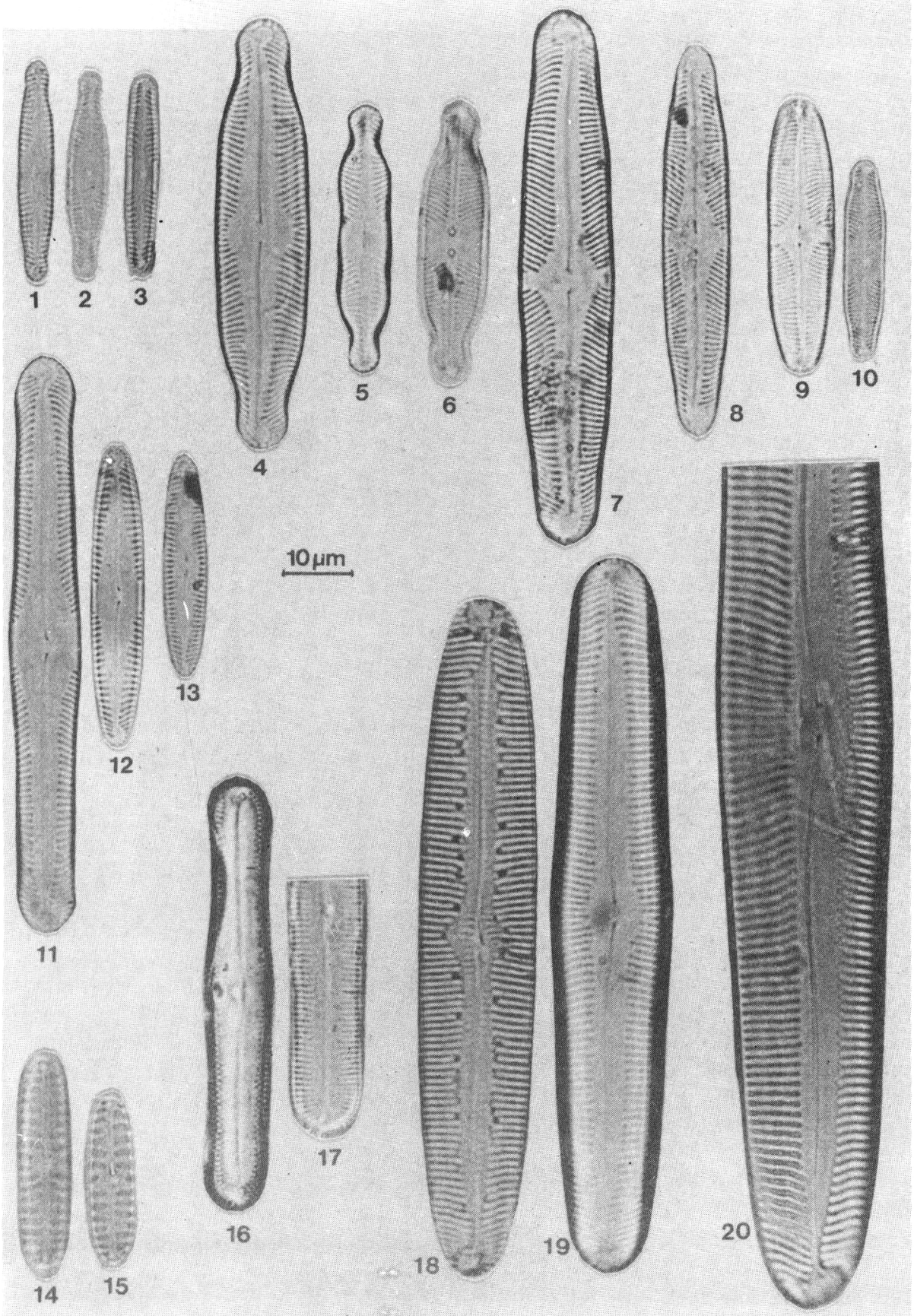

Figura 5. 1. Pinnularia subcapitata - 2. P. subcapitata var. hilseana. - 3. P. subcapitata. - 4. P. biceps. - 5. P. mesolepta. - 6. P. lundii. - 7. P. divergens. - 8-10. P. microstauron.-11-13. P. gibba. - 14-15. P. borealis. - 16-17. P. acrosphaeria. - 18-20. P. viridis. 
de anchura; 11 estrías cada $10 \mu \mathrm{m}$.

Localidades: A $(3,31,4,5,6), \mathrm{H}$ (excepto $32)$, L (2, 3, 4, 62, 8), M (1, 2, 31), G (2), D (1, 11).

\section{Navicula laevissima Kütz.}

$\left(\mathrm{n}=5, \sum \mathrm{H}=5\right)$. (Fig. 4: 35)

Localidades: H (1, 31, 5), L (2, 3), G (3).

Navicula lanceolata (Ag.) Ehr.

(n=102, $\sum H=227$ ). (Fig. 4: 9, 10, 17)

Una de las peculiaridades de esta especie, la presencia de una media estría o la falta de una estría completa, no siempre es observable.

Localidades: Excepto L42 y L61

Navicula menisculus Schumann

$\left(\mathrm{n}=1, \sum \mathrm{H}=1\right)$. (Fig. 4: 18)

Localidad: A (3).

Navicula nyassensis $O$. Müller

$(\mathrm{n}=2, \Sigma \mathrm{H}=2$ ). (Fig. 4: 36)

La hemos encontrado en aguas salobreñas, claras y deficitarias en sustancias nitrogenadas.

Localidades: L (41 ), M (3).

\section{Navicula oblonga Kütz.}

$(\mathrm{n}=3, \Sigma \mathrm{H}=4)$. (Fig. 4: 24)

Localidades: H (31), G.

Navicula perpusilla Grun.

$(\mathrm{n}=3, \Sigma \mathrm{H}=3$ ). (Fig. 4: 37)

Localidades: A (1), L $(5,8)$.

Navicula placentula (Ehr.) Grun.

$\left(\mathrm{n}=1, \sum \mathrm{H}=1\right)$. (Fig. 4: 28)

Localidad: A (4).

Navicula pseudolanceolata Lange-B.

$(\mathrm{n}=3, \Sigma \mathrm{H}=5)$. (Fig. 4: 21)

En aguas de elevada conductividad y no polutas.

Medidas: Valvas con 38-39 $\mu \mathrm{m}$ de longitud y

7-8 $\mu \mathrm{m}$ de anchura; 11 estrías cada $10 \mu \mathrm{m}$.

Localidades: H $(4,6), \mathrm{G}(2)$.

Navicula pseudonivalis Bock

$(\mathrm{n}=2, \Sigma \mathrm{H}=2$ ). (Fig. 4: 32)

Aparece en aguas de conductividad modera-

da y con bajo grado de turbidez.

Medidas: Valvas de $17 \mu \mathrm{m}$ de longitud y 5-6 $\mu \mathrm{m}$ de anchura.

Localidades: L (42), D (11).

Navicula pseudotuscula Hust.

$(\mathrm{n}=1, \Sigma \mathrm{H}=1)$. (Fig. 4: 38)

Tan sólo observamos un ejemplar de rafe sinuoso y tamaño menor de lo habitual $(23 \times 9 \mu \mathrm{m})$. Localidad: $\mathbf{G}(2)$.

Navicula pupula Kütz.

$(\mathrm{n}=58, \Sigma \mathrm{H}=87$ ). (Fig. $4: 34)$

La variedad capitata Hust., se presentó en un par de ocasiones.

Localidades: A (excepto 6), $\mathrm{H}$ (excepto 1, 4 y 61), L (3, 4, 42, 5, 61, 62, 7, 8), M, G, D (1).

Navicula pygmaea Kütz.

$(\mathrm{n}=6, \Sigma \mathrm{H}=7$ ). (Fig. 4: 29)

Localidades: A (4), H (3), M (2), G (2).

\section{Navicula radiosa Kütz.}

(Fig. $4: 16$ )

Localidades: A $(1,2,3,4,5,6), \mathrm{H}(1,31,32$, 5, 6, 61), L (5, 61), M (1, 21, 3, 31), G.

Navicula rhynchocephala Kütz.

$\left(\mathrm{n}=1078, \sum \mathrm{H}=204\right)$. (Fig. 4: 8)

Localidades: Excepto H61 y M31.

Navicula rostellata Kütz.

$\left(\mathrm{n}=27, \sum \mathrm{H}=47\right)$. (Fig. 4: 6)

Localidades: A $(31,4,5,51,6), \mathrm{H}(3,31,41$, 5, 6, 61, 7), L (2 , 3,4, 7, 8), M (3), D (12).

Navicula sclesvicensis Grun.

$\left(\mathrm{n}=2, \sum \mathrm{H}=3\right)$. (Fig. 4: 7)

Se presenta en condiciones similares a las de

N. pseudolanceolata.

Localidades: H (6), G (2).

Navicula trivialis Lange-B.

$\left(\mathrm{n}=27, \sum \mathrm{H}=47\right.$ ). (Fig. 4: 19)

Localidades: A $(3,4,6), \mathrm{H}(1,2,21,3,31,32$, $4,41,6), \mathrm{L}(2,4,42,62,7,8), \mathrm{M}(1,21,3,31), \mathrm{G}$ (2), D (11).

Navicula viridula Kütz.

$(\mathrm{n}=6, \Sigma \mathrm{H}=8)$. (Fig. 4: 5)

Localidades: A (4), M (1, 2, 3). 


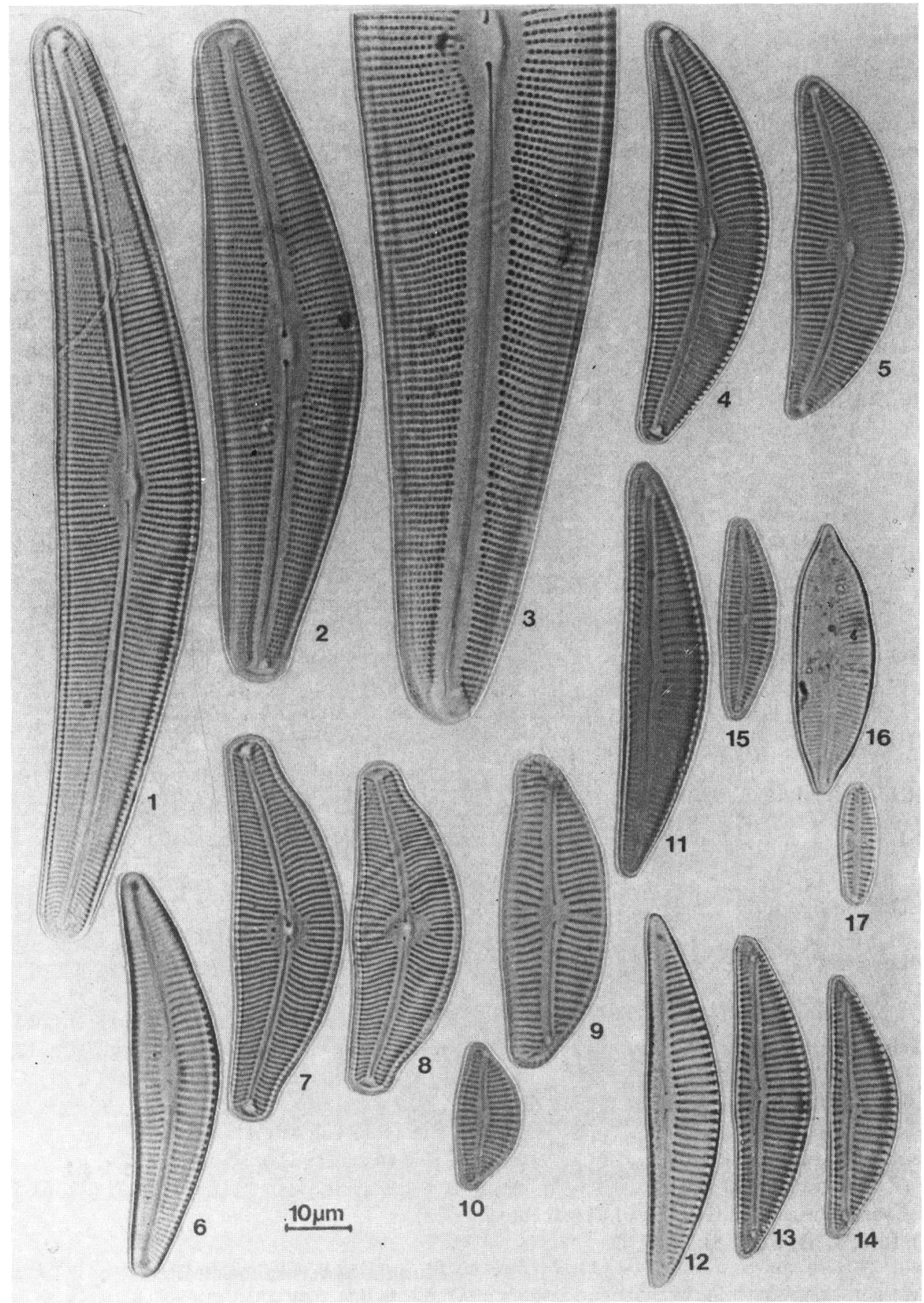

Figura 6. 1. Cymbella lanceolata. - 2-3. C. aspera. - 4-5. C. cistula. - 6. C. parva. - 7-8. C. tumida. - 9. C. prostata. - 10. C. caespitosa.-11.-C. turgida.-12-14. C. minuta.-15. C. leptoceros.-16. C. heteropleura var.minor. - 17. C. sinuata fma. ovata. 
Neidium affine (Ehr.) $\mathrm{Cl}$.

( $\mathrm{n}=30, \Sigma \mathrm{H}=31)$. (Fig. 3: 12, 14)

La variedad amphirhynchus (Ehr.)Cl. (fig. 14 ), fue la más observada; en Aldehuela de Yeltes apareció, además, con su fma. undulata Hust. (fig. 12).

Localidades: A (1, 2, 4, 5), $\mathrm{H}(2,21,32,4,41$, $6,7), \mathrm{L}(1,2,3,4,7,8), \mathrm{M}(1,21,3,31), \mathrm{G}, \mathrm{D}(11)$.

Neidium dubium (Ehr.) $\mathrm{Cl}$.

$(\mathrm{n}=3, \Sigma \mathrm{H}=3$ ). (Fig. 3: 13)

Localidades: G (2), D (11).

Neidium iridis (Ehr.) $\mathrm{Cl}$.

$(\mathrm{n}=5, \Sigma \mathrm{H}=5)$. (Fig. 3: 15)

Localidades: A (1), H (6), L (5, 62 ), G (2).

Nitzschia acicularis W. Sm.

( $\mathrm{n}=16, \Sigma \mathrm{H}=25$ )

Localidades: A $(2,4,51,6), \mathrm{H}(2,21,31,32$, 41, 5, 7), L (3), G (3), D (11).

Nitzschia acuta Hantzsch

$(\mathrm{n}=11, \Sigma \mathrm{H}=17)$. (Fig. 8: 5-6)

Localidades: $\mathrm{H}(3,31,32,4,41,5), \mathrm{M}(21,3)$, G (2), D (1, 11).

Nitzschia apiculata (Greg.) Grun.

$(\mathrm{n}=2, \Sigma \mathrm{H}=2$ ). (Fig. 8: 16)

Localidad: G (2)

Nitzschia dissipata (Kütz.) Grun.

$(\mathrm{n}=20, \Sigma \mathrm{H}=35$ )

Localidades: A $(2,3,6), \mathrm{H}(1,3,31,4,41,5$, 6, 61, 7), L (2, 3), M (2, 3, 31), G (2), D (1, 11).

Nitzschia dubia W. Sm.

(n= 5, $\Sigma H=9$ ). (Fig. 8: 15)

Localidades: H (31), L (8), M (1, 31), G (2).

Nitzschia hantzschiana Rabh.

$\left(\mathrm{n}=68, \sum \mathrm{H}=132\right)$. (Fig. 8: 7)

Localidades: A $(3,31,5,51,6), \mathrm{H}$ (excepto 2

y 21$), \mathrm{L}(2,3,61,62,7,8), \mathrm{M}, \mathrm{G}, \mathrm{D}$.

Nitzschia hungarica Grun.

( $\mathrm{n}=9, \Sigma \mathrm{H}=13$ )

Localidades: H $(31,4)$, M $(2,31)$, G, D (11).

Nitzschia linearis W. Sm. (n $=79, \Sigma \mathrm{H}=156$ ). (Fig. 8: 9-10)

Localidades: Excepto H5, L5, L8 y D12

Nitzschia obtusa W Sm. var. scalpelliformis Grun. $(\mathrm{n}=1, \Sigma \mathrm{H}=2)$. (Fig. 8: 14)

Localidad: A (4).

\section{Nitzschia palea gr.}

(Fig. 8: 8)

La dificultad que existe para distinguir a mil aumentos las especies N. palea (Kütz.) W. Sm. en especial, su variedad debilis (Kütz.) Grun.—de $N$. paleacea Grun. es muy elevada, a pesar de que sea un conjunto muy frecuente en todas las aguas estudiadas.

Nitzschia parvula Lewis

(n=1, $\sum \mathrm{H}=1$ ). (Fig. 8: 13)

Localizada en aguas claras de poca conductividad

Medidas: Valvas de $30 \times 8 \mu \mathrm{m} ; 8$ fíbulas cada $10 \mu \mathrm{m}$.

Localidad: A (6).

Nitzschia sigmoidea (Ehr.) W. Sm. $(\mathrm{n}=15, \Sigma \mathrm{H}=18)$. (Fig. 8: 1)

Localidades: $\mathrm{H}(3,31,4,41,5), \mathrm{M}(21,3,31)$,

G, D $(1,11)$.

Nitzschia sinuata W. Sm.

$(\mathrm{n}=9, \Sigma \mathrm{H}=10)$. (Fig. 8: 3-4)

Localidades: A (3), H (3, 6, 61, 7), L (2,3).

Nitzschia tryblionella Hantzsch

$(\mathrm{n}=7, \Sigma \mathrm{H}=10)$. (Fig. 8: 11-12)

Suele estar acompañada, aunque en poca cantidad, de la variedad subsalina Grun. (fig. 12).

Localidades: $\mathrm{H}(3,31,41), \mathrm{M}(2,21), \mathrm{G}(2)$.

Nitzschia valdestriata Aleem \& Hust.

( $\mathrm{n}=10, \Sigma \mathrm{H}=11)$. (Fig. 8: 2 )

Localidades: A $(1,31,51), \mathrm{H}(32,6), \mathrm{D}(1$, 12).

Pinnularia acrosphaeria Bréb.

$(\mathrm{n}=3, \Sigma \mathrm{H}=3)$. (Fig. 5: 16-17)

Localidades: H (1), L (4), G (3).

Pinnularia biceps Greg.

$(\mathrm{n}=17, \Sigma \mathrm{H}=17)$. (Fig. 5: 4) 




Figura 7. 1. Amphora ovalis, vista conectiva. - 2. A. ovalis, vista valvar.- 3. A. pediculus. - 4. A. veneta, vista conectiva. - 5. -A. veneta, vista valvar. - 6. Gomphoneis olivacea. - 7. Gomphonema acuminatum. - 8. G. acuminatum var. coronata. - 9. G. acuminatum var. elongata - 10. G. acuminatum. - 11. G. acuminatum var. brebissonii. - 12-13. G. augur. - 14. G. lanceolatum var. insignis. - 15. G. intricatum. 16. G. constrictum.- 17. G. constrictum var. capitata.- 18. G. angustatum.- 19. G. angustatum var. producta.- 20. G. gracile.- 21-24. Gomphoneis clevei. 
Localidades: A (1, 2), H (1, 21, 3, 4), L (1, 2 , 42, 8), M (31), G (2), D (1).

Pinnularia borealis Ehr.

$(\mathrm{n}=21, \Sigma \mathrm{H}=23$ ). (Fig. 5: 14-15)

Localidades: A $(1,31,4,5,6), \mathrm{H}(2,3,31,32$, $41,5,6), \mathrm{L}(42,5,6,61,7), \mathrm{G}(2)$.

Pinnularia brebissonnii (Kütz.) Rabh.

$(\mathrm{n}=3, \Sigma \mathrm{H}=3)$

Localidades: H (3), M (31), G (2).

Pinnularia divergens W. Sm.

$\left(\mathrm{n}=8, \sum \mathrm{H}=8\right)$. (Fig. 5: 7)

Localidades: A (1), H (1, 2, 21), L $(4,8)$.

Pinnularia gibba Ehr.

$(\mathrm{n}=42, \Sigma \mathrm{H}=49$ ). (Fig. 5: 11-13)

Localidades: A (menos 6), $\mathrm{H}$ (menos $21,4 \mathrm{y}$ 61), L (1, 2, 4, 5, 6, 61, 62, 7, 8), M (2, 21, 3, 31).

Pinnularia lundii Hust.

$(\mathrm{n}=5, \Sigma \mathrm{H}=5)$. (Fig. 5: 6)

Frecuenta las mismas aguas que Achnanthes peregalli.

Aunque nuestros ejemplares sean algo mayores $(44 \times 10 \mu \mathrm{m})$ de lo habitual y cuenten con más estrías ( 15 cada $10 \mu \mathrm{m}$ ), por mejores razones (peculiar capitación, rafe recto hasta el centro, fisura terminal oblicua, etc.), caben en esta especie.

Localidades: A (1, 2), H (21), L (4, 7).

Pinnularia mesolepta (Ehr.) W. Sm.

$(\mathrm{n}=14, \Sigma \mathrm{H}=23$ ). (Fig. $5: 5)$

Localidades: H (1, 6), L (4, 7, 8), G (2).

Pinnularia microstauron (Ehr.) $\mathrm{Cl}$.

$(\mathrm{n}=19, \Sigma \mathrm{H}=23)$. (Fig. $5: 8-10)$

Localidades: A $(1,31,4,5,6), \mathrm{H}(2,21,4,6$, 7), $\mathrm{L}(1,3,5,6,7,8)$.

Pinnularia subcapitata Greg.

$\left(\mathrm{n}=16, \sum \mathrm{H}=32\right)$. (Fig. $\left.5: 1-3\right)$

La variedad hilseana (Janisch) O. Müller (fig.

2), es una acompañante asidua del tipo.

Localidades: A (1, 4), H $(31,6), \mathrm{L}$ (excepto 3$)$.

Pinnularia viridis (Nitzsch) Ehr.

$(\mathrm{n}=52, \Sigma \mathrm{H}=67)$. (Fig. 5: 18-20)

Algunos ejemplares mayúsculos no serían difíciles de incluir en P. maior (Kütz.) Cl. (fig. 20).
Localidades: A (1, 2, 3, 31, 4), H (1, 2, 21, 3, $31,4,41,6$ ), L (excepto 2), M (excepto 2), G.

Rhoicosphenia curvata (Kütz.) Grun $(\mathrm{n}=51, \Sigma \mathrm{H}=92)$. (Fig. 3: 10-11)

Localidades: A $(5,51,6), \mathrm{H}(1,3,31,4,41,5$, 6, 7), L (2, 3, 8), M (excepto 1), G, D (1, 11).

Rhopalodia gibba (Ehr.) O. Müller $\left(n=26, \sum H=43\right)$. (Fig. 9: 7-8)

Casi siempre aparece con su variedad ventricosa (Ehr.) Grun.

Localidades: A $(4,6), \mathrm{H}(3,31,32,4,41,5,6$, 7), L (1, 2), G (2), D (12).

Rhopalodia gibberula (Ehr.) O. Müller var. vanheurcki $O$. Müller.

$\left(\mathrm{n}=1, \sum \mathrm{H}=1\right)$. (Fig. 9: 10)

Localidad: $\mathrm{G}(2)$.

Rhopalodia parallela (Grun.) O. Müller

$(\mathrm{n}=3, \Sigma \mathrm{H}=4)$. (Fig. 9: 9)

Localidades: H $(31,32), \mathrm{G}(2)$

Stauroneis anceps Ehr.

$(\mathrm{n}=20, \Sigma \mathrm{H}=20)$. (Fig. 3: 3-4)

La fma. gracilis (Ehr.) Cl. apareció en contadas ocasiones.

Localidades: A $(1,6), \mathrm{H}(2,21,3,31,4,5,6)$,

$\mathrm{L}(1,4,5,61,62,7), \mathrm{G}(3)$.

Stauroneis phenicentheron Ehr.

$\left(\mathrm{n}=34, \sum \mathrm{H}=38\right)$. (Fig. 3, figs $\left.1-2\right)$

La figura 2 puede asimilarse a la fma. gracilis (Ehr.) Cl., que es lo más común de esta especie en las aguas estudiadas.

Localidades: A (1, 3), H (1, 2, 21, 31, 32, 4, 5,

6), L $(1,4,5,61,62,7,8), \mathrm{M}(21,3,31)$, G.

Stauroneis smithii Grun.

$(\mathrm{n}=5, \Sigma \mathrm{H}=6)$. (Fig. 3: 5)

Localidades: L (2), M (21), G.

Stephanodiscus astraea (Ehr.) Grun.

$\left(\mathrm{n}=5, \sum \mathrm{H}=12\right)$. (Fig. 1: 6)

Localidades: D.

Surirella biseriata Bréb.

$(\mathrm{n}=25, \Sigma \mathrm{H}=26)$. (Fig. 10: 1)

Localidades: A (1, 2, 3, 31, 5), H (6, 7), L (2, 


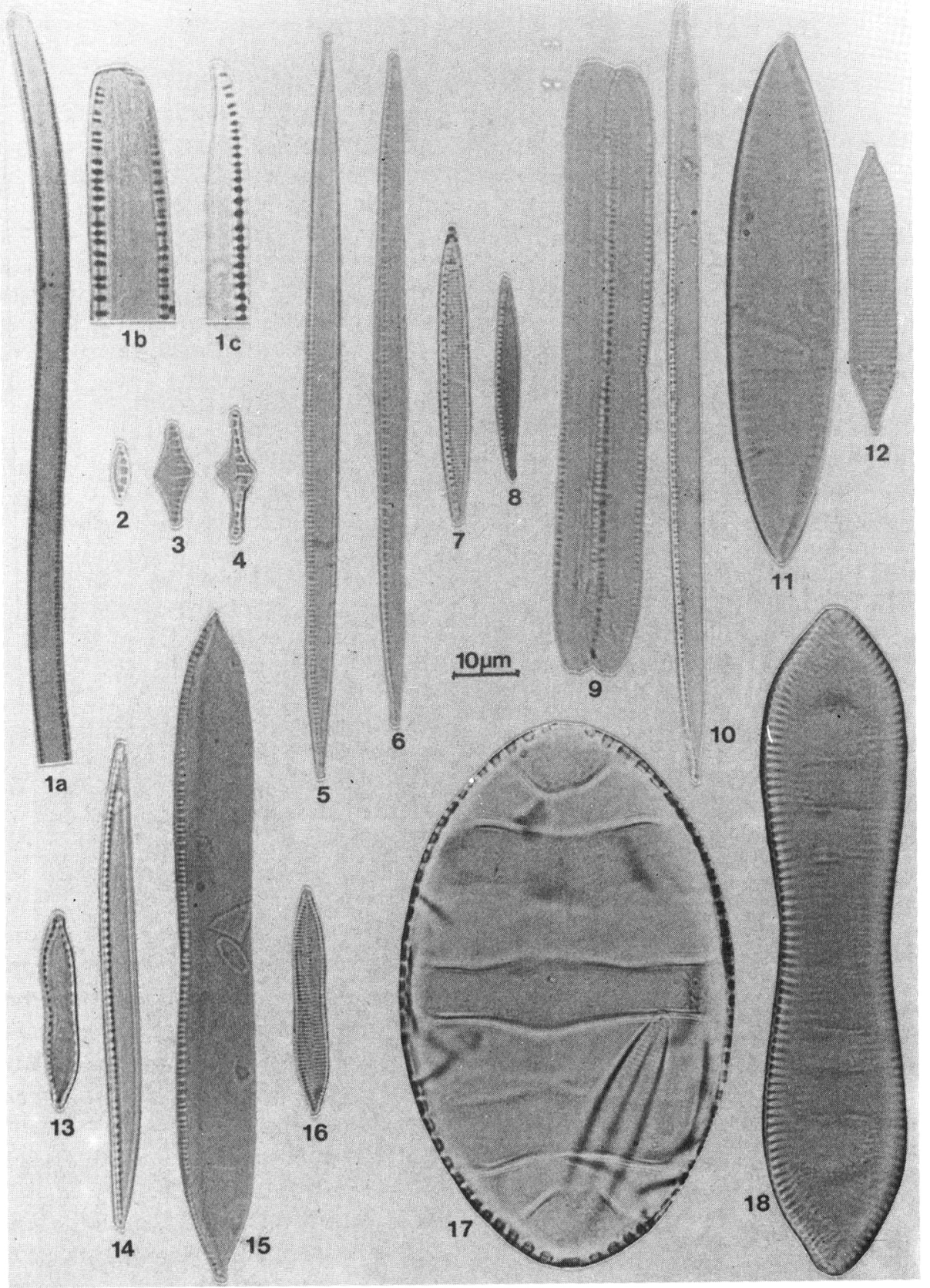

Figura 8. 1a. Nitzschia sigmoidea (x 400). - 1b. N. sigmoidea, vista conectiva. - 1c. N. sigmoidea, vista valvar. - 2. N. valdestriata. - 3-4. N. sinuata. - 5-6. N. acuta. - 7. N. hantzschiana. - 8. ¿ N. paleacea ?. 9-10. N. linearis. - 11. N. tryblionella. - 12. N. tryblionella var. subsalina. - 13. N. parvula. - 14. N. obtusa var. scalpelliformis. - 15. N. dubia. - 16. N. apiculata. - 17. Cymatopleura elliptica. - 18. C. solea. 
$3,4,41,5,61,62,8), \mathrm{M}(3)$.

Surirella elegans Ehr.

$(\mathrm{n}=9, \Sigma \mathrm{H}=11)$. (Fig. 10:2)

3), G (2).

Localidades: A (3, 31), H (5, 6), L (2, 5), M (1,

\section{Surirella ovalis Bréb.}

$(\mathrm{n}=25, \Sigma \mathrm{H}=32)$. (Fig. 10: 3-5)

Localidades: A (31), H (2, 21, 3, 31, 4), M , G, D (11).

Surirella ovata Kütz.

$(\mathrm{n}=102, \Sigma \mathrm{H}=154)$. (Fig. 10: 7-10)

De las múltiples variedades que se hacen de esta especie, contamos con pinnata W. Smith (fig. 7) y apiculata W. Smith (fig. 10).

Localidades: Excepto H61 y L61

Surirella striatula Turpin

$(\mathrm{n}=1, \Sigma \mathrm{H}=1)$. (Fig. 10: 6)

Vimos un ejemplar de esta gran especie y, además, lejos de sus emplazamientos ordinarios.

Localidad: H (21)

\section{Synedra acus Kütz.}

$(\mathrm{n}=61, \Sigma \mathrm{H}=130)$

Localidades: A (2, 3, 4, 5, 51, 6), H, L (1, 2, 3, 4, 61), M (excepto 3), G, D $(1,12)$.

Synedra capitata Ehr.

( $\mathrm{n}=14, \Sigma \mathrm{H}=23$ ). (Fig. 2: 25) (2), G.

Localidades: A (3), H (3, 32, 4, 5, 6), L (1), M

Synedra parasitica W. Sm.

$(\mathrm{n}=8, \Sigma \mathrm{H}=16)$. (Fig. 2: 30-31)

Siempre está acompañada de su variedad subconstricta Grun. (fig. 30).

Localidades: A (4), H (5, 6), L (2), M (3), G (2), D (1).

\section{Synedra pulchella Kütz.}

$(\mathrm{n}=57, \Sigma \mathrm{H}=121)$. (Fig. 2: 28)

Localidades: A, H (excepto 21), L (2, 6, 8), M

(3), D (1).

\section{Synedra rumpens Kütz.}

$(\mathrm{n}=54, \Sigma \mathrm{H}=115)$

Localidades: A, H (excepto 32), L (excepto
6), M (2), D (11).

Synedra tabulata Ag.

( $\mathrm{n}=18, \sum \mathrm{H}=32$ ). (Fig. 2: 29)

Localidades: H (3, 32, 4,6), L (3), G , D (1).

Synedra ulna (Nitzsch) Ehr.

( $\mathrm{n}=148, \Sigma \mathrm{H}=471$ ). (Fig. 2: $26-27$ además del tipo se encuentran la variedad biceps (Kuetz.) von Schönfldt y, como era de esperar en una especie tan extendida, formas intermedias.

Localidades: Todas las estudiadas.

Synedra vaucheriae Kütz.

( $\mathrm{n}=118, \Sigma \mathrm{H}=338$ ). (Fig. $2: 32$ )

Hay una serie continua de morfotipos hacia la variedad capitellata Grun.

Localidades: Todas las estudiadas

Tabellaria fenestrata (Lyngb.) Kütz. ( $\mathrm{n}=27, \sum \mathrm{H}=28$ ). (Fig. 1: 14-15)

Localidades: A (excepto 4), H (1, 32, 4, 5, 6, 7), L (3), D (11, 12).

Tabellaria flocculosa (Roth) Kütz. $\left(\mathrm{n}=71, \sum \mathrm{H}=122\right.$ ). (Fig. 1: 12-13)

Localidades: A (excepto 4), H (1, 2, 21, 3, $31,32,41,5,6)$, L, D $(1,12)$.

\section{DISCUSIÓN}

Entre los táxones relacionados, Achnanthes peregalli, Cymbella heteropleura, Gomphonema acuminatum var. elongata, Navicula clementis, Navicula cuspidata var. heribaudi, Navicula hungarica, Navicula nyassensis, Navicula pseudolanceolata, Navicula pseudonivalis, Navicula pseudotuscula, Navicula sclesvicensis, Nitzschia parvula, Nitzschia valdestriata y Pinnularia lundii, tras la consulta de la recopilación de Alvarez Cobelas y Estevez García (1982) y de diversos trabajos posteriores (Varela, 1982; Aboal y Llimona, 1984; Sabater, 1984, Sánchez Castillo, 1984 y 1988; Aboal, 1986 y 1989; Sabater et al. 1987; Santos Valdés 1987 ; etc.) suponen primeras citas para nuestro país. 


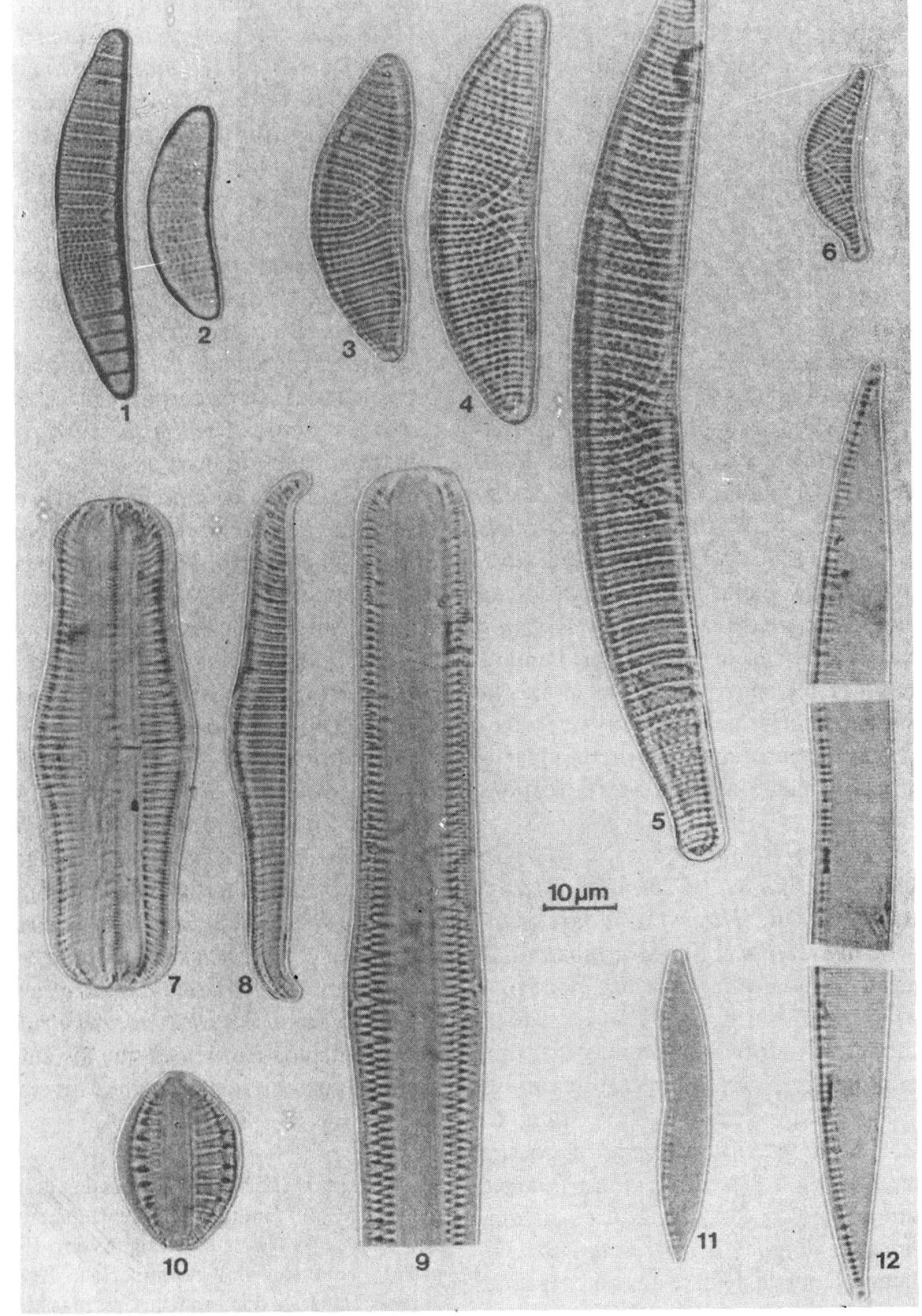

Figura 9. 1-2. Epithemia zebra. - 3-5. E. turgida. - 6. E. sorex. - 7. Rhopalodia gibba, vista conectiva. 8. $R$. gibba, vista valvar.- 9. $R$. parallela, vista conectiva. - 10. $R$. gibberula var. vanheurcki. - 11. Hantzschia amphioxys. - 12. H. amphioxys var. maior. 
Si relacionamos el conjunto de datos biológicos con el conjunto de datos físico-químicos podemos señalar una serie de grupos bióticos que responden a otros tantos requerimientos ecológicos. De esta forma, hemos observado que Achnanthes hungarica, Navicula gregaria, $N$. viridula, Nitzschiadubia, $N$. hungarica y N. triblyonella se presentan en aguas de elevado residuo salino; en éstas son frecuentes además, Cocconeis pediculus, Cymatopleura solea, Gomphoneis olivacea, Navicula cuspidata, N. dicephala, N. pygmaea, Nitzschia acicularis y Surirella ovalis. Cuando las disponibilidades de oxígeno aumentan, la composición de las poblaciones se enriquecen en Cymbella prostrata, Navicula bacillum, $N$. deccusis, N. goppertiana, N. gracilis, Nitzschia dissipata, N. sigmoidea, Stauroneis smithii, Synedra parasitica y Surirella elegans. Las aguas más propicias para el desarrollo de estas especies son las de los ríos Almar y Guareña ya que discurren por las llanuras miocenas del NE provincial. Las prácticas agrícolas deben jugar un papel muy importante en la composición química del agua, elevando significativamente las cantidades de fosfatos y sulfatos.

Amphipleura pellucida, Cymbella lanceolata, C. parva, Diploneis ovalis, Epithemia sp pl, Navicula rostellata, Pinnularia mesolepta y Synedra tabulata se encuentran en aguas mineralizadas, poco oxigenadas, ricas en cloruros y de bajos contenidos en fosfatos y sulfatos. Con más oxígeno, incrementan sus poblaciones Achnanthes clevei, Cymbella caespitosa, C. cistula, C. turgida, Cymatopleura elliptica, Diatoma vulgare, Diploneis puella, Eunotia lunaris, Navicula oblonga, Fragilaria brevistriata, Gomphonema augur y Synedra capitata. De modo general, puede decirse que son especies de las aguas que circulan por la penillanura salmantina; en épocas lluviosas suelen mantener frecuencias medias o bajas en las cuencas del Almar y Guareña.
En aguas claras, bien ventiladas, poco o nada mineralizadas y muy pobres en sales viven Achnanthes peregalli, Ceratoneis arcus, Cyclotella stelligera, Eunotia diodon, Eunotia veneris, Gomphoneis clevei, Navicula cari var. angusta, Tabellaria fenestrata, T. flocculosa, Pinnularia lundii y Surirella biseriata. Estos medios también son los preferidos de Achnanthes exigua var. heterovalvata, Cymbella sinuata fma. ovata, Diatoma elongatum, Fragilaria pinnata, Navicula laevissima, Neidium iridis, Nitzschia sinuata y Synedra pulchella. Cuando disminuye la presión de oxígeno incorporan más individuos especies como Cymbella heteropleura, Diatoma hiemale var. mesodon, Eunotia pectinalis, Pinnularia biceps, $P$. borealis, $P$. divergens, $P$. microstauron y $P$. subcapitata. Son, en su conjunto, especies que buscan los tramos superiores de las cuencas de los ríos Alagón, Huebra-Yeltes y Agueda.

Achanthes lanceolata, Cocconeis placentula, Cyclotella meneghiniana, Cymbella minuta, Gomphonema acuminatum, G. angustatum, G. constrictum, Melosira varians, Navicula rhynchocephala, Nitzchia linearis, Surirella ovata, Synedra ulna y $S$. vaucheriae, no son especies muy exigentes ya que raras veces faltaron en las preparaciones. Amphoraveneta, Cymbella tumida, Fragilaria capucina, F. construens, Frustulia vulgaris, Gomphonema gracile, Melosira ambigua, Meridion circulare y Pinnularia viridis, aunque son menos frecuentes que las anteriores, también aparecieron en medios diversos.

AGRADECIMIENTOS. Damos las gracias a la Doctora María Candida P. Gil del Departamento de Biología de la Universidad de Aveiro (Portugal) por la revisión del manuscrito. Asímismo, agradecemos a la doctora Rita Carabias Martínez y a su equipo de trabajo la cesión de los datos químicos que aparecen en este trabajo. 


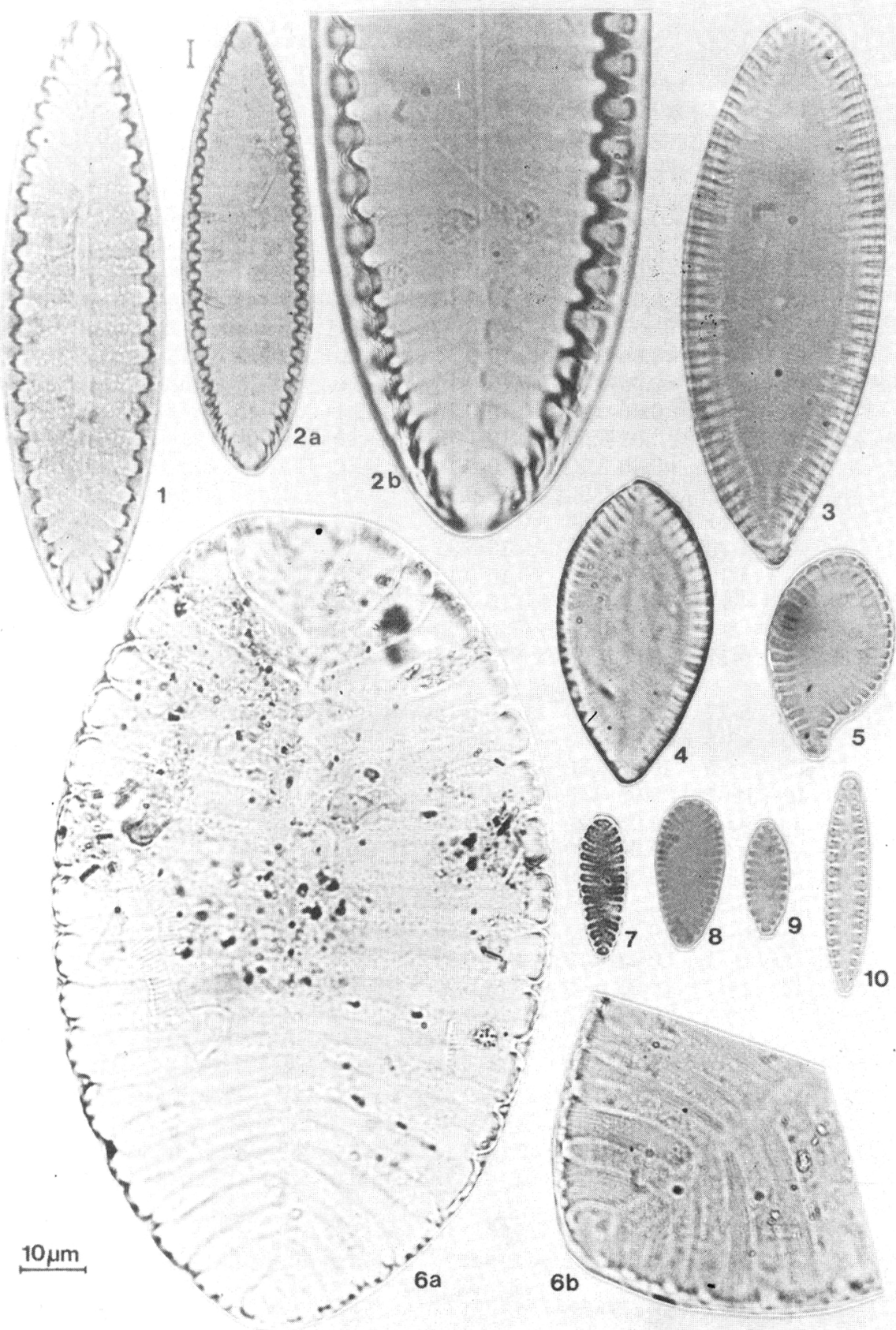

Figura 10. 1. Surirella biseriata. - 2a. S. elegans (x 400). - 2b. S. elegans, detalle del pseudorafe. - 3-4. S. ovalis. - 5. S. ovalis, defecto habitual en las poblaciones cuantiosas. - 6a. S. striatula. - 6b. S. striatula, detalle de la estriación. - 7. S. ovata var. pinnata. - 8. S. ovata. - 9. S. ovata var. angustata. - 10. S. ovata var. apiculata. 


\begin{tabular}{|c|c|c|c|c|c|c|c|c|c|c|}
\hline Estación & $\mathrm{pH}$ & $\begin{array}{l}\mathrm{T}^{\mathrm{a}} \\
{ }^{\circ} \mathrm{C}\end{array}$ & $\begin{array}{c}\mathrm{DQO} \\
\mathrm{mg} / \mathrm{l}\end{array}$ & $\begin{array}{c}\mathrm{NH}_{3}{ }^{+} \mathrm{NH}_{4}^{+} \\
\mathrm{mg} / \mathrm{l}\end{array}$ & $\begin{array}{l}\mathrm{NO}_{2}^{-} \\
\mathrm{mg} / \mathrm{l}\end{array}$ & $\begin{array}{l}\mathrm{NO}_{3}^{-} \\
\mathrm{mg} / \mathrm{l}\end{array}$ & $\begin{array}{c}\text { Conductivida } \\
\mu \mathrm{S} / \mathrm{cm}\end{array}$ & $\begin{array}{ll}\text { lad } & \mathrm{PO}_{4}{ }^{3-} \\
& \mathrm{mg} / \mathrm{l} \\
\end{array}$ & $\begin{array}{c}\mathrm{Cl}^{-} \\
\mathrm{mg} / \mathrm{l}\end{array}$ & $\begin{array}{r}\mathrm{SO}^{2} \\
\mathrm{mg} / 1\end{array}$ \\
\hline A1 & 6.2 & 13 & $<3-5$ & $<0.06-0.20$ & $<0.10-0.10$ & $<5-6.7$ & $24-34$ & $<15-19$ & $<0.2-2.9$ & $<8-<8$ \\
\hline A2 & 6.4 & 13 & $<3-15$ & $<0.06-0.30$ & $<0.10-0.10$ & $<5-5.0$ & $23-54$ & $<15-40$ & $<0.2-14.5$ & $<8.14$ \\
\hline A3 & 6.7 & 15 & $<3-13$ & $<0.06-0.90$ & $<0.10-0.13$ & $<5-11.0$ & $30-91$ & $90-213$ & $1.3-4.4$ & $<8-11$ \\
\hline A31 & - & - & $<3-6$ & $0.08-0.20$ & $<0.10-0.13$ & $<5-11.0$ & 55-114 & $20-119$ & $1.1-6.3$ & $<8-37$ \\
\hline A4 & 7.9 & 17 & 4-14 & $<0.06-0.30$ & $0.10-0.16$ & $<5-11.0$ & $75-156$ & $38-120$ & $4.2-12.0$ & $<8-25$ \\
\hline A5 & 7.1 & 14 & $<3-4$ & $0.08-0.70$ & $<0.10-0.13$ & $<5-15.3$ & $60-112$ & $24-125$ & $1.6-6.3$ & $<8-37$ \\
\hline A51 & - & - & $<3-6$ & $<0.06-0.20$ & $<0.10-0.11$ & $<5-5.0$ & $65-107$ & $24-38$ & $2.0-6.5$ & $11-29$ \\
\hline A6 & 7.3 & 15 & $<3-6$ & $<0.06-1.40$ & $<0.10-0.16$ & $<5-5.4$ & $69-141$ & $30-51$ & $2.2-6.4$ & $<8-24$ \\
\hline H1 & 7.9 & 14 & $4-17$ & $<0.06-0.70$ & $<0.10-0.33$ & $<5-11.0$ & $59-247$ & $<15-65$ & $7.5-30.6$ & $<8-45$ \\
\hline H2 & 7.3 & 14 & $3-58$ & $<0.06-0.20$ & $<0.10-0.16$ & $<5-6.0$ & $48-79$ & $<15-625$ & $0.6-5.5$ & $<8-10.3$ \\
\hline H21 & - & - & $3-6$ & $<0.06-0.20$ & $<0.10-0.10$ & $<5-13.1$ & $47-55$ & $<15-31$ & $<0.2-5.1$ & $<8-8$ \\
\hline H3 & 7.9 & 14 & $3-95$ & $0.08-5.60$ & $<0.10-1.73$ & $<5-5.1$ & $1 \quad 199-700$ & $25-120$ & $10.4-47.0$ & $13-46$ \\
\hline H31 & - & - & $3-10$ & $<0.06-0.20$ & $<0.10-0.10$ & $<5-5.0$ & 105-186 & $<15-100$ & $1.0-13.3$ & $12-24$ \\
\hline H32 & - & - & $8-12$ & $<0.06-0.90$ & $<0.10-0.10$ & $<5-5.0$ & 231-453 & $<15-288$ & $15.3-57.1$ & $8-17$ \\
\hline H4 & 8.2 & 14 & $8-11$ & $<0.06-0.80$ & $<0.10-0.16$ & $<5-5.0$ & 219-336 & $20-455$ & $8.6-38.2$ & $<8-21$ \\
\hline H41 & - & - & $3-10$ & $<0.06-0.80$ & $<0.10-0.33$ & $<5-5.0$ & $82-224$ & $60-275$ & $6.6-20.1$ & $10-19$ \\
\hline H5 & 7.5 & 14 & $<3-10$ & $0.08-0.90$ & $<0.10-0.19$ & $<5-5.0$ & $82-158$ & $25-88$ & $7.8-13.1$ & $<8-15$ \\
\hline H6 & 7.7 & 14 & $4-11$ & $0.15-0.80$ & $<0.10-0.10$ & $<5-5.0$ & $92-205$ & $<15-170$ & $8.5-18.9$ & $<8-21$ \\
\hline H61 & - & - & $5-8$ & $0.08-0.60$ & $<0.10-0.10$ & $<5-5.0$ & 101-170 & $<15-160$ & $8.5-12.6$ & $<8-13$ \\
\hline H7 & 8.0 & 15 & $6-11$ & $<0.06-1.00$ & $<0.10-0.22$ & $<5-5.0$ & $100-220$ & 23-144 & $9.3-22.8$ & $<8-35$ \\
\hline L1 & 7.8 & 10 & $5-27$ & $<0.06-1.10$ & $<0.10-0.16$ & $<5-5.0$ & 12 & $<15-31$ & 6.8 & $<8-48$ \\
\hline L2 & 7.3 & 10 & $<3-6$ & $<0.06-0.40$ & $<0.10-0.11$ & $<5-5.0$ & $77-139$ & $<15-37$ & $4.3-54.4$ & $<8-8$ \\
\hline L3 & 7.2 & 10 & $<3-6$ & $<0.06-0.30$ & $<0.10-0.10$ & $<5-5.0$ & $67-112$ & $15-44$ & $0.7-6.2$ & $8-14$ \\
\hline L4 & 6.6 & 10 & $<3-5$ & $<0.06-0.40$ & $<0.10-010$ & $<5-11.0$ & $26-64$ & $20-79$ & $<0.2-4.3$ & $<8-<8$ \\
\hline L41 & - & - & $<3-5$ & $<0.06-0.20$ & $<0.10-0.10$ & $<5-6.7$ & $22-54$ & $28-62$ & $<0.2-3.4$ & $<8-8$ \\
\hline L42 & - & - & $<3-6$ & $<0.06-0.20$ & $<0.10-0.10$ & $<5-11.0$ & $19-34$ & $<15-38$ & $<0.2-3.2$ & $<8-<8$ \\
\hline L5 & 6.6 & 9 & $<3-3$ & $<0.06-0.30$ & $<0.10-0.10$ & $<5-5.0$ & $15-58$ & $<15-37$ & $<0.2-1.0$ & $<8-<8$ \\
\hline L6 & 6.9 & 10 & $10-32$ & $0.58-1.50$ & $0.22-0.44$ & $<5-5.0$ & $90-207$ & $236-575$ & $<0.2-78.0$ & $8-27$ \\
\hline L61 & - & - & $6-11$ & $0.30-1.40$ & $0.25-0.34$ & $<5-5.0$ & $84-140$ & $150-438$ & $0.9-11.6$ & $<8-25$ \\
\hline L62 & - & - & $5-11$ & $0.08-1.10$ & $0.16-0.39$ & $<5-5.8$ & $77-120$ & $94-450$ & $1.9-9.9$ & $<8-16$ \\
\hline L7 & 6.9 & 8 & $5-10$ & $<0.06-0.60$ & $<0.10-0.25$ & $<5-5.0$ & 54-105 & $114-350$ & $0.6-6.9$ & $<8-20$ \\
\hline L8 & 6.9 & 9 & $5-9$ & $0.07-0.70$ & $<0.10-0.18$ & $<5-5.0$ & $61-90$ & $53-274$ & $0.4-5.7$ & $<8-14$ \\
\hline M1 & 7.8 & 12 & $8-15$ & .68 & .30 & $<5-8.9$ & $9 \quad 180$ & 80 & 3.9 & $13-68$ \\
\hline M2 & 7.9 & 12 & $<3-8$ & $0.08-0.98$ & $<0.10-0.57$ & $<5-5.0$ & م $194-839$ & $150-540$ & $14.4-66.1$ & $19-159$ \\
\hline M21 & - & - & $4-11$ & $0.07-1.20$ & $<0.10-0.44$ & $<5-5.0$ & 232-608 & $68-825$ & $19.0-63.0$ & $20-42$ \\
\hline M3 & 7.9 & 10 & $7-17$ & $0.08-0.40$ & $<0.10-0.16$ & $<5-5.7$ & $7 \quad 259-486$ & $256-560$ & $17.3-36.9$ & $11-25$ \\
\hline M31 & - & - & $10-20$ & $0.70-9.28$ & $<0.10-0.78$ & $<5-9.0$ & $0243-6502$ & $219-1830$ & $14.0-92.8$ & $13-31$ \\
\hline G2 & 7.9 & 10 & & .50 & & $<5.0$ & & & & $<8-23$ \\
\hline G3 & 7.8 & 10 & $5-13$ & $<0.06-0.12$ & $<0.10-0.26$ & $<5-9.0$ & $0 \quad 623-738$ & $25-215$ & $32.7-43.1$ & $19-37$ \\
\hline D1 & 8.2 & 16 & $<3-6$ & $<0.06-0.50$ & .25 & $<5-25.0$ & $0 \quad 24$ & $68-289$ & 10.1 & $29-61$ \\
\hline D11 & - & - & $<3-9$ & $0.18-0.40$ & $0.11-0.25$ & $<5-7.0$ & $0 \quad 247-364$ & 94-185 & $9.8-18.6$ & $35-40$ \\
\hline D12 & - & - & $<3-10$ & $0.20-0.30$ & $0.10-0.36$ & $<5-16.5$ & $5 \quad 270-384$ & $68-225$ & $10.6-20.6$ & $31-67$ \\
\hline
\end{tabular}

Tabla 2. Rango de variación obtenido de las principales variables físico-químicas del agua para cada una de las estaciones de muestreo. Hacen excepción los valores de $\mathrm{pH}$ y temperatura pues únicamente se tomaron en la primera campaña. 


\section{BIBLIOGRAFÍA}

ABOAL, M. -1986-. Flora algal de la rambla del río Tinajón (río Segura), Murcia; S.E. de España. Limnética, 2: 103-108.

ABOAL, M. -1989-. Flora algal del río Benamor (Cuenca del Segura, SE de España). Limnética, 5:1-11.

ABOAL, M. y X. LLIMONA -1984-. Aportación al conocimiento de la flora del río Mula, Murcia. S.E. de España. Limnética, 1:141-147.

ALMEIDA RINO, J. y M.C. GIL -1987-. Diatomáceas do rio Cértima - Estudo preliminar. Rev. Biol. Univ. Aveiro, vol. 1: 53-103.

ALVAREZ COBELAS, M. y A. ESTEVEZ GARCÍA -1982-. Catálogo de las algas continentales españolas I : Diatomophyceae Rabenhorst 1864. Lazaroa, 4: 269-285.

BOURRELY, P. -1968-. Les algues d'eau douce. Iniciation à la systématique 2. Ed. N. Boubée \& Cie. Paris.

CAMBRA, J. -1987-. Flora et végétation algologiques des eaux épicontinentales de l'a Reserve Naturelle des vallés d'Ordesa (Pyrénées aragonaises). Candollea, 42:475-490.

DESCY, J.P. -1979-. A new approach to water quality estimation using Diatoms. Nova Hedwigia, 64:305-323.

GALLEGO MARTÍN, F.,SÁNCHEZ ANTA, $M^{a}$. A., y R. GARCIA RIO.- Variabilidad morfológica y ecología de Gomphoneis clevei (Fricke) M. C. Gil en los ríos salmantinos. Aceptado para su publicación en Anales del Jardín Botánico de Madrid.

GERMAIN , H. -1981-. Flore des diatomées. Eaux douces et saumâtres du Masif Armoricain et des contrées voisines d'Europe occidentale. Société Nouvelle des éditions Boubée. Paris, $444 \mathrm{p}$.

HUSTEDT, F. -1930-. Bacillariophyta (Diatomeae), in Die Susswasserflora Mitteleuropas, 10: 468 p. Koenigstein/W. Germany.

MARGALEF, R. - 1948-. Las asociaciones de algas en las aguas dulces de pequeño volumen del NE de España. Vegetatio, 1: 258-284.

PATRICK, R. \& C. W. REIMER, -1975-. The diatoms of the United States Monographs of the Academy of Natural Sciences of Philadelphia, núm. 13, vol. 2, part. 1:213 p.
SABATER, S., SABATER, F. \& X. TOMAS, -1987-. Water quality and diatoms communities in two catalan rivers (N.E. Spain). Wat. Res. vol., 21, núm. 8:901-911.

SÁNCHEZ CASTILLO, P.M. -1984-. Estudio ficológico de la vegetación reófila de Sierra Nevada (Granada). Limnética, 1:136-144.

SÁNCHEZ CASTILLO, P.M. -1988-. Aproximación al estudio ficológico de Sierra Nevada. Monogr. Fl. y Veg. Béticas, 3: 29-40.

SANTOS VALDÉS, M. -1987-. Catálogo de las algas continentales en unas charcas pluviales de Villanueva del Arbol (León, España). Act. VI Simp. Nac. Bot. Cript.,: 155-164.

VARELA, M. -1982-. Adiciones a la flora de diatomeas de agua dulce de Galicia. IV Simposi de Botànica Criptogàmica. Barcelona.

Aceptado para su publicación en Junio de 1992

Dirección de los autores: Departamento de Biología Vegetal (Biología General). Facultad de Biología. Universidad de Salamanca. Plaza de la Merced. CP. 37008. Salamanca. 